\title{
CONVEX INTEGRATION FOR THE MONGE-AMPÈRE EQUATION IN TWO DIMENSIONS
}

\author{
MARTA LEWICKA AND MOHAMMAD REZA PAKZAD
}

\begin{abstract}
This paper concerns the questions of flexibility and rigidity of solutions to the MongeAmpère equation which arises as a natural geometrical constraint in prestrained nonlinear elasticity. In particular, we focus on anomalous i.e. "flexible" weak solutions that can be constructed through methods of convex integration à la Nash \& Kuiper and establish the related $h$-principle for the Monge-Ampère equation in two dimensions.
\end{abstract}

\section{Contents}

1. Introduction.

1.1. The weak determinant Hessian.

1.2. Convex integration for the Monge-Ampère equation in two dimensions. 3

1.3. Rigidity versus flexibility. 4

1.4. Notation. 5

1.5. Acknowledgments. 5

2. The $\mathcal{C}^{1}$ approximations - preliminary results.

3. The $\mathcal{C}^{1}$ approximations - a proof of Theorem 2.1 9

4. The $\mathcal{C}^{1, \alpha}$ approximations - a proof of Theorem 1.1, preliminary results and some heuristics towards the proof of Theorem 1.2 .

5. The $\mathcal{C}^{1, \alpha}$ approximations - a 'step' and a 'stage' in a proof of Theorem 1.2. 17

6. The $\mathcal{C}^{1, \alpha}$ approximations - a proof of Theorem 1.2 22

7. Rigidity results for $\alpha>2 / 3$ - a proof of Theorem 1.3 . 26

\begin{tabular}{ll} 
References & 29. \\
\hline
\end{tabular}

\section{Introduction.}

In this paper we study the $\mathcal{C}^{1, \alpha}$ solutions to the Monge-Ampère equation in two dimensions:

$$
\operatorname{Det} \nabla^{2} v:=-\frac{1}{2} \operatorname{curl} \operatorname{curl}(\nabla v \otimes \nabla v)=f \quad \text { in } \Omega \subset \mathbb{R}^{2} .
$$

Our results concern the dichotomy of "rigidity vs. flexibility", in the spirit of the analogous results and techniques appearing in the contexts of: the low co-dimension isometric immersion problem [37, 30, 3, 4, 10, and the Onsager's conjecture for Euler equations [42, 12, 13, 9, 15.

In the first, main part of the paper we show that below the regularity threshold $\alpha<1 / 7$, the very weak $\mathcal{C}^{1, \alpha}(\bar{\Omega})$ solutions to $(1.1)$ as defined below, are dense in the set of all continuous functions (see Theorems 1.1 and 1.2). These flexibility statements are a consequence of the convex integration $h$-principle, that is a method proposed in [17] for solving certain partial differential relations and that turns out to be applicable to our setting of the Monge-Ampère equation as well. 
Here, we directly adapt the iteration method of Nash and Kuiper [37, 30], in order to construct the oscillatory solutions to 1.1 . 1 1

In the second part of the paper we prove that the same class of very weak solutions fails the above flexibility in the regularity regime $\alpha>2 / 3$. Our results are parallel with those concerning isometric immersions [3, 10, 38, Euler equations [9, 15], Perona-Malik equation [25, 26], the active scalar equation [20], and should also be compared with results on the regularity of Sobolev solutions to the Monge-Ampère equation [38, 41, 31, 24] whose study is important in the context of nonlinear elasticity and with the rigidity results for the Monge-Ampère functions [22, 23].

1.1. The weak determinant Hessian. Let $\Omega \subset \mathbb{R}^{2}$ be an open set. Given a function $v \in$ $W_{l o c}^{1,2}(\Omega)$, we define its very weak Hessian (denoted by $\mathcal{H}_{2}^{*}$ in $[21,16]$ ) as:

$$
\text { Det } \nabla^{2} v=-\frac{1}{2} \operatorname{curl} \operatorname{curl}(\nabla v \otimes \nabla v) \text {, }
$$

understood in the sense of distributions. A straightforward approximation argument shows that if $v \in W_{l o c}^{2,2}$ then $L_{l o c}^{1}(\Omega) \ni \operatorname{Det} \nabla^{2} v=\operatorname{det} \nabla^{2} v$ a.e. in $\Omega$, where $\nabla^{2} v$ stands for the Hessian matrix field of $v$. We also remark that this notion of the very weak Hessian is distinct from the distributional Hessian $\operatorname{Det} \nabla^{2} v=\operatorname{Det} \nabla(\nabla v)$ (denoted by $\mathcal{H} u$ in [21, 16]), that is defined through the distributional determinant Det:

$$
\operatorname{Det} \nabla \psi=-\operatorname{div}\left(\psi_{2} \nabla^{\perp} \psi_{1}\right)=\partial_{2}\left(\psi_{2} \partial_{1} \psi_{1}\right)-\partial_{1}\left(\psi_{2} \partial_{2} \psi_{1}\right) \quad \text { for } \quad \psi=\left(\psi_{1}, \psi_{2}\right) \in W^{1,4 / 3}\left(\Omega, \mathbb{R}^{2}\right) .
$$

Contrary to the distributional Hessian, the very weak Hessian is not continuous with respect to the weak topology. Indeed, an example of a sequence $v_{n} \in W^{1,2}(\Omega)$ is constructed in [21], where $\operatorname{Det} \nabla^{2} v=-1$ while $v_{n}$ converges weakly to 0 . One consequence of the proof of our Theorem 1.1 below is that $\operatorname{Det} \nabla^{2}$ is actually weakly discontinuous everywhere in $W^{1,2}(\Omega)$ (see Corollary 6.2).

Here is our first main result:

Theorem 1.1. Let $f \in L^{7 / 6}(\Omega)$ on an open, bounded, simply connected $\Omega \subset \mathbb{R}^{2}$. Fix an exponent:

$$
\alpha<\frac{1}{7}
$$

Then the set of $\mathcal{C}^{1, \alpha}(\bar{\Omega})$ solutions to $\left.\overline{1.1}\right)$ is dense in the space $\mathcal{C}^{0}(\bar{\Omega})$. More precisely, for every $v_{0} \in \mathcal{C}^{0}(\bar{\Omega})$ there exists a sequence $v_{n} \in \mathcal{C}^{1, \alpha}(\bar{\Omega})$, converging uniformly to $v_{0}$ and satisfying:

$$
\text { Det } \nabla^{2} v_{n}=f \quad \text { in } \Omega \text {. }
$$

When $f \in L^{p}(\Omega)$ and $p \in\left(1, \frac{7}{6}\right)$, the same result is true for any $\alpha<1-\frac{1}{p}$.

In order to better understand Theorem 1.1, we point out a connection between the solutions to (1.1) and the isometric immersions of Riemannian metrics, motivated by a study of nonlinear elastic plates. Since on a simply connected domain $\Omega$, the kernel of the differential operator curl curl consists of the fields of the form sym $\nabla w$, a solution to (1.1) with the vanishing right hand side $f \equiv 0$ can be characterized by the criterion:

$$
\exists w: \Omega \rightarrow \mathbb{R}^{2} \quad \frac{1}{2} \nabla v \otimes \nabla v+\operatorname{sym} \nabla w=0 \quad \text { in } \Omega .
$$

\footnotetext{
${ }^{1}$ We remark that the recent work of De Lellis, Inaunen and Szekelyhidi [1] showed that the flexibility exponent $\frac{1}{7}$ can be improved to $\frac{1}{5}$ in the case of the isometric immersion problem in 2 dimensions. We expect similar improvement to be possible also in the present case of equation 1.1 ; this will be investigated in our future work.
} 
The equation in (1.3) can be seen as an equivalent condition for the following 1-parameter family of deformations, given through the out-of-plane displacement $v$ and the in-plane displacement $w$ (albeit with different orders of magnitude $\varepsilon$ and $\varepsilon^{2}$ ):

$$
\phi_{\varepsilon}=i d+\varepsilon v e_{3}+\varepsilon^{2} w: \Omega \rightarrow \mathbb{R}^{3}
$$

to form a 2nd order infinitesimal isometry (bending), i.e. to induce the change of metric on the plate $\Omega$ whose 2 nd order terms in $\varepsilon$ disappear:

$$
\left(\nabla \phi_{\varepsilon}\right)^{T} \nabla \phi_{\varepsilon}-\mathrm{Id}_{2}=o\left(\varepsilon^{2}\right) .
$$

In this context, we take the cue about Theorem 1.1 from the celebrated work of Nash and Kuiper [37, 30], where they show the density of co-dimension one $\mathcal{C}^{1}$ isometric immersions of Riemannian manifolds in the set of short mappings. Since we are are now dealing with the 2nd order infinitesimal isometries rather than the exact isometries, the classical metric pull-back equation:

$$
y^{*} g_{e}=h,
$$

for a mapping $y$ from $(\Omega, h)$ into $\mathbb{R}^{3}$ equipped with the standard Euclidean metric $g_{e}$, is replaced by the compatibility equation of the tensor $T(v, w)=\frac{1}{2} \nabla v \otimes \nabla v+\operatorname{sym} \nabla w$ with a matrix field $A_{0}$ that satisfies: - curl curl $A_{0}=f$ :

$$
T(v, w)=A_{0}
$$

Note that there are many potential choices for $A_{0}$, for example one may take $A_{0}(x)=\lambda(x) \operatorname{Id}_{2}$ with $\Delta \lambda=-f$ in $\Omega$. Again, equation (1.4) states precisely that the metric $\left(\nabla \phi_{\varepsilon}\right)^{T} \nabla \phi_{\varepsilon}$ agrees with the given metric $h=\mathrm{Id}_{2}+2 \varepsilon^{2} A_{0}$ on $\Omega$, up to terms of order $\varepsilon^{2}$. The Gauss curvature $\kappa$ of the metric $h$ satisfies:

$$
\kappa(h)=\kappa\left(\operatorname{Id}_{2}+2 \varepsilon^{2} A_{0}\right)=-\varepsilon^{2} \operatorname{curl} \operatorname{curl} A_{0}+o\left(\varepsilon^{2}\right),
$$

while $\kappa\left(\left(\nabla \phi_{\varepsilon}\right)^{T} \nabla \phi_{\varepsilon}\right)=-\varepsilon^{2} \operatorname{curl} \operatorname{curl}\left(\frac{1}{2} \nabla v \otimes \nabla v+\operatorname{sym} w\right)+o\left(\varepsilon^{2}\right)$, so the problem (1.1) can also be interpreted as seeking for all appropriately regular out-of-plane displacements $v$ that can be matched, by a higher order in-plane displacement perturbation $w$, to achieve the prescribed Gauss curvature $f$ of $\Omega$, at its highest order term.

In this paper, similarly as in the isometric immersion case, we show that solutions to (1.4) are ample. We design a scheme inspired by the work of Nash and Kuiper, which pushes a "short infinitesimal isometry", i.e. a couple $\left(v_{0}, w_{0}\right)$ such that $T\left(v_{0}, w_{0}\right)<A_{0}$, towards an exact solution to (1.4) in successive small steps. Note that both $y^{*} g_{e}=(\nabla y)^{T} \nabla y$ and the term $\nabla v \otimes \nabla v$ in $T(v, w)$ have a quadratic structure, which is crucial in the analysis of [37, 30] and also of this paper. Here, not only the presence of the linear term $\operatorname{sym} \nabla w$ in $T(u, w)$ does not destroy the adaptation of the Nash-Kuiper scheme, but it actually allows for this construction to work.

1.2. Convex integration for the Monge-Ampère equation in two dimensions. As we will see in section 4, Theorem 1.1 follows easily from the statement of our next main result:

Theorem 1.2. Let $\Omega \subset \mathbb{R}^{2}$ be an open and bounded domain. Let $v_{0} \in \mathcal{C}^{1}(\bar{\Omega}), w_{0} \in \mathcal{C}^{1}\left(\bar{\Omega}, \mathbb{R}^{2}\right)$ and $A_{0} \in \mathcal{C}^{0, \beta}\left(\bar{\Omega}, \mathbb{R}_{\text {sym }}^{2 \times 2}\right)$, for some $\beta \in(0,1)$, be such that:

$$
\exists c_{0}>0 \quad A_{0}-\left(\frac{1}{2} \nabla v_{0} \otimes \nabla v_{0}+\operatorname{sym} \nabla w_{0}\right)>c_{0} \operatorname{Id}_{2} \quad \text { in } \bar{\Omega} .
$$


Then, for every exponent $\alpha$ in the range:

$$
0<\alpha<\min \left\{\frac{1}{7}, \frac{\beta}{2}\right\}
$$

there exist sequences $v_{n} \in \mathcal{C}^{1, \alpha}(\bar{\Omega})$ and $w_{n} \in \mathcal{C}^{1, \alpha}\left(\bar{\Omega}, \mathbb{R}^{2}\right)$ which converge uniformly to $v_{0}$ and $w_{0}$, respectively, and which satisfy:

$$
A_{0}=\frac{1}{2} \nabla v_{n} \otimes \nabla v_{n}+\operatorname{sym} \nabla w_{n} \quad \text { in } \bar{\Omega} .
$$

The above result is the Monge-Ampère analogue of [10, Theorem 1], where the authors improved on the Nash-Kuiper method to obtain higher regularity within the flexibility regime. In our paper, we adapt the same methods to our problem.

Convex integration was originally developed by Gromov [17] to deal with finding weak solutions of a differential inclusion $L u(x) \in K$ in $\Omega$, by investigating certain classes of sub-solutions, e.g. functions $u$ that satisfy $L u(x) \in \operatorname{conv} K$ where the original constraint set $K$ is replaced by its appropriate convex hull conv $K$. Under specific circumstances, it leads to establishing the density of very weak solutions, satisfying $L u \in L^{\infty}(\Omega)$, in the set of sub-solutions, and in case the constraint set is a continuum the regularity might be improved to $L u \in \mathcal{C}^{0}(\Omega)$.

Recently, these methods were applied in the context of fluid dynamics and yielded many interesting results for the Euler equations. In [12], De Lellis and Székelyhidi proved existence of weak solutions with bounded velocity and pressure, their non-uniqueness and the existence of energydecreasing solutions. In [13], using iteration methods à la Nash-Kuiper, the same authors proved existence of continuous periodic solutions of the 3-dimensional incompressible Euler equations, which dissipate the total kinetic energy. These results are to be contrasted with [9, 15], where it was shown that $\mathcal{C}^{0, \alpha}$ solutions of the Euler equations are energy conservative if $\alpha>1 / 3$. There have been several improvements of [12, 13] since, towards a possible proof of the Onsager's conjecture which puts the Hölder regularity threshold for the energy conservation of the weak solutions to the Euler equations at $\mathcal{C}^{0,1 / 3}[18,19,5,6,7,8$. The stationary incompressible Euler equation has been studied in [8] where the existence of bounded anomalous solutions have been proved. The authors indicate that in 2 dimensions, the relaxation set corresponding to the appropriate subsolutions is smaller than in the case of the evolutionary equations. In this context, we noticed a connection between our reformulation of the Monge-Ampère equation and the steady state Euler equation, which lead to our modest Corollary 4.1 .

In this paper we use a direct iteration method to construct exact solutions of (1.1). The recasting of the statement and the proof in the language of convex integration might shed more light on the structure of the Monge-Ampère equation, but it would not improve the results and therefore we do not address this task. We note, however, that constructing Lipschitz continuous piecewise affine approximating solutions to $(1.6)$ for $A_{0} \equiv 0$ is quite straightforward and could be used to prove a convex integration density result via the Baire category method as was done in 12 for the Euler equations (see also Figure 2.1 and the corresponding explanation).

1.3. Rigidity versus flexibility. The flexibility results obtained in view of the $h$-principle are usually coupled with the rigidity results for more regular solutions. Rigidity of isometric immersions of elliptic metrics for $\mathcal{C}^{1, \alpha}$ isometries [3, 12] with $\alpha>2 / 3$, or the energy conservation of weak solutions of the Euler equations for $\mathcal{C}^{0, \alpha}$ solutions with $\alpha>1 / 3$, are results of this type. For the Monge-Ampère equations, we recall two recent statements regarding solutions with Sobolev regularity: following the well known unpublished work by Šverák [41, we proved in [31] that if 
$v \in W^{2,2}(\Omega)$ is a solution to 1.1 with $f \in L^{1}(\Omega)$ and $f \geq c>0$ in $\Omega$, then in fact $v$ must be $\mathcal{C}^{1}$ and globally convex (or concave). On the other hand, if $f=0$ then [38] likewise $v \in \mathcal{C}^{1}(\Omega)$ and $v$ must be developable (see also [22, 23, 24]). A clear statement of rigidity is still lacking for the general $f$, as is the case for isometric immersions, where rigidity results are usually formulated only for elliptic [10] or Euclidean metrics [38, 34, 24].

In this paper, we prove the rigidity properties of solutions to (1.1) in the Hölder regularity context when $f \equiv 0$. Namely, we prove:

Theorem 1.3. Let $\Omega \subset \mathbb{R}^{2}$ be an open, bounded domain and let:

$$
\frac{2}{3}<\alpha<1 \text {. }
$$

If $v \in \mathcal{C}^{1, \alpha}(\bar{\Omega})$ is a solution to $\mathcal{D}$ et $\nabla^{2} v=0$ in $\bar{\Omega}$, then $v$ must be developable. More precisely, for all $x \in \Omega$ either $v$ is affine in a neighbourhood of $x$, or there exists a segment $l_{x}$ joining $\partial \Omega$ on its both ends, such that $\nabla v$ is constant on $l_{x}$.

We also announce the following parallel rigidity result $f \geq c>0$, that will be the subject of the forthcoming paper [32]:

Theorem 1.4. Let $\Omega \subset \mathbb{R}^{2}$ be an open, bounded domain and let:

$$
\frac{2}{3}<\alpha<1 \text {. }
$$

If $v \in \mathcal{C}^{1, \alpha}(\bar{\Omega})$ is a solution to $\operatorname{Det} \nabla^{2} v=f$ in $\bar{\Omega}$, where $f$ is a positive Dini continuous, then $v$ is convex. In fact, it is also an Alexandrov solution to $\operatorname{det} \nabla^{2} v=f$ in $\Omega$.

In proving Theorem 1.3 , we use a commutator estimate for deriving a degree formula in Proposition 7.1. Similar commutator estimates are used in [9] for the Euler equations and in [10] for the isometric immersion problem; this is not surprising, since the presence of a quadratic term plays a major role in all three cases, allowing for the efficiency of the convex integration and iteration methods. Let us also mention that it is still an open problem which value of $\alpha$ is the critical value for the rigidity-flexibility dichotomy, and it is conjectured to be $1 / 3,1 / 2$ or $2 / 3$.

1.4. Notation. By $\mathbb{R}_{s y m}^{2 \times 2}$ we denote the space of symmetric $2 \times 2$ matrices, and by $\mathbb{R}_{s y m,>}^{2 \times 2}$ we denote the cone of symmetric, positive definite $2 \times 2$ matrices. The space of Hölder continuous functions $\mathcal{C}^{k, \alpha}(\bar{\Omega})$ consists of restrictions of functions $f \in \mathcal{C}^{k, \alpha}\left(\mathbb{R}^{2}\right)$ to $\Omega \subset \mathbb{R}^{2}$. Then, the $\mathcal{C}^{k}(\bar{\Omega})$ norm of such restriction is denoted by $\|f\|_{k}$, while its Hölder norm $\mathcal{C}^{k, \alpha}(\bar{\Omega})$ is $\|f\|_{k, \alpha}$. By $C>0$ we denote a universal constant which is independent of all parameters, unless indicated otherwise.

1.5. Acknowledgments. The authors would like to thank Camillo De Lellis for discussions about this problem. This project is based upon work supported by, among others, the National Science Foundation. M.L. was partially supported by the NSF grants DMS-0846996 and DMS-1406730. M.R.P. was partially supported by the NSF grant DMS-1210258. A part of this work was completed while the authors visited the Forschungsinstitut für Mathematik at ETH (Zurich, Switzerland). The institute's hospitality is gratefully acknowledged.

\section{The $\mathcal{C}^{1}$ APproximations - PRELIMINARY RESUlts.}

In this and the next section we prove a weaker version of the result in Theorem 1.2 . Namely: 
Theorem 2.1. Let $\Omega \subset \mathbb{R}^{2}$ be an open and bounded domain. Let $v_{0} \in \mathcal{C}^{\infty}(\bar{\Omega}), w_{0} \in \mathcal{C}^{\infty}\left(\bar{\Omega}, \mathbb{R}^{2}\right)$ and $A_{0} \in \mathcal{C}^{\infty}\left(\bar{\Omega}, \mathbb{R}_{\text {sym }}^{2 \times 2}\right)$ be such that:

$$
\exists c_{0}>0 \quad A_{0}-\left(\frac{1}{2} \nabla v_{0} \otimes \nabla v_{0}+\operatorname{sym} \nabla w_{0}\right)>c_{0} \operatorname{Id}_{2} \quad \text { in } \bar{\Omega} .
$$

Then there exist sequences $v_{n} \in \mathcal{C}^{1}(\bar{\Omega})$ and $w_{n} \in \mathcal{C}^{1}\left(\bar{\Omega}, \mathbb{R}^{2}\right)$ which converge uniformly to $v_{0}$ and $w_{0}$ respectively, and which satisfy:

$$
A_{0}=\frac{1}{2} \nabla v_{n} \otimes \nabla v_{n}+\operatorname{sym} \nabla w_{n} \quad \text { in } \bar{\Omega} .
$$

We start with a series of preliminary lemmas whose details we provide for the sake of completeness. The first lemma is an observation in convex integration, pertaining to solving an appropriate differential inclusion to be used for constructing the 1-dimensional oscillatory perturbations in $v_{n}$ and $w_{n}$. As always, $C>0$ is a universal constant, independent of all parameters, in particular independent of the function $a$ below.

Lemma 2.2. Let $a \in \mathcal{C}^{\infty}(\bar{\Omega})$ be a nonnegative function on an open and bounded set $\Omega \subset \mathbb{R}^{2}$. There exists a smooth 1-periodic field $\Gamma=\left(\Gamma_{1}, \Gamma_{2}\right) \in \mathcal{C}^{\infty}\left(\bar{\Omega} \times \mathbb{R}, \mathbb{R}^{2}\right)$ such that the following holds for all $(x, t) \in \bar{\Omega} \times \mathbb{R}$ :

$$
\begin{aligned}
& \Gamma(x, t+1)=\Gamma(x, t), \\
& \frac{1}{2}\left|\partial_{t} \Gamma_{1}(x, t)\right|^{2}+\partial_{t} \Gamma_{2}(x, t)=a(x)^{2},
\end{aligned}
$$

together with the uniform bounds:

$$
\begin{aligned}
& \left|\Gamma_{1}(x, t)\right|+\left|\partial_{t} \Gamma_{1}(x, t)\right| \leq C a(x), \quad\left|\Gamma_{2}(x, t)\right|+\left|\partial_{t} \Gamma_{2}(x, t)\right| \leq C a(x)^{2}, \\
& \left|\nabla_{x} \Gamma_{1}(x, t)\right| \leq C|\nabla a(x)|, \quad\left|\nabla_{x} \Gamma_{2}(x, t)\right| \leq C|a(x)||\nabla a(x)| .
\end{aligned}
$$

Proof. Firstly, note that there exists a smooth 1-periodic function $\gamma \in \mathcal{C}^{\infty}\left(\mathbb{R}, \mathbb{R}^{2}\right)$, such that for all $t \in \mathbb{R}$ there holds:

$$
\begin{aligned}
& \gamma(t+1)=\gamma(t), \quad \int_{0}^{1} \gamma(t) \mathrm{d} t=(0,0) \\
& \gamma(t) \in P:=\left\{\left(s_{1}, s_{2}\right) \in \mathbb{R}^{2} ; \frac{1}{2} s_{1}^{2}+s_{2}=1,\left|s_{1}\right| \leq 2\right\} .
\end{aligned}
$$

Existence of $\gamma$ is a consequence of the fundamental lemma of convex integration, since the intended average $(0,0)$ lies in the convex hull of the parabola $P$ (see Figure 2.1). Indeed, one can take:

$$
\gamma(t)=(2 \cos (2 \pi t),-\cos (4 \pi t)) \in P .
$$

It is now enough to ensure that $\partial_{t} \Gamma_{1}=a(x) \gamma_{1}(x)$ and $\partial_{t} \Gamma_{2}=a(x)^{2} \gamma_{2}(x)$ to obtain (2.3). Namely:

$$
\Gamma_{1}(x, t)=\frac{a(x)}{\pi} \sin (2 \pi t), \quad \Gamma_{2}(x, t)=-\frac{a(x)^{2}}{4 \pi} \sin 4 \pi t .
$$

We see directly that the bounds in 2.4 hold.

To compare with the problem of isometric immersions, note that in that context, a 1-dimensional convex integration lemma is similarly proved in [42, Figure2, p. 11], where instead of a parabola, the constraint set consists of a full circle.

We will also need a special case of [10, Lemma 3] about decomposition of positive definite symmetric matrices into rank-one matrices. 


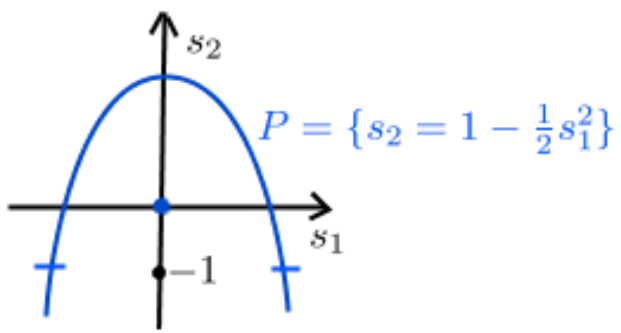

Figure 2.1. The parabola $P$ in the $1 \mathrm{~d}$ convex integration problem of Lemma 2.2 .

Lemma 2.3. There exists a sufficiently small constant $r_{0}>0$ such that the following holds. For

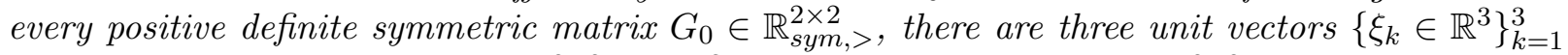
and three linear functions $\left\{\Phi_{k}: \mathbb{R}_{\text {sym }}^{2 \times 2} \rightarrow \mathbb{R}_{k=1}^{3}\right.$, such that: for any $G \in \mathbb{R}_{\text {sym }}^{2 \times 2}$ we have

$$
\forall G \in \mathbb{R}_{\text {sym }}^{2 \times 2} \quad G=\sum_{k=1}^{3} \Phi_{k}(G) \xi_{k} \otimes \xi_{k},
$$

and that each $\Phi_{k}$ is strictly positive on the ball $B\left(G_{0}, r\left(G_{0}\right)\right) \subset \mathbb{R}_{\text {sym }}^{2 \times 2}$ with radius $r\left(G_{0}\right)=\frac{r_{0}}{\left|G_{0}^{-1 / 2}\right|^{2}}$.

Proof. 1. First, assume that $G_{0}=\mathrm{Id}_{2}$. Set:

$$
\zeta_{1}=\frac{1}{\sqrt{12}}(2+\sqrt{2},-2+\sqrt{2}), \quad \zeta_{2}=\frac{1}{\sqrt{12}}(-2+\sqrt{2}, 2+\sqrt{2}), \quad \zeta_{3}=\frac{1}{\sqrt{2}}(1,1) .
$$

In order to check that the following matrices form a basis of the 3 -dimensional space $\mathbb{R}_{\text {sym }}^{2 \times 2}$ :

$\zeta_{1} \otimes \zeta_{1}=\frac{1}{12}\left[\begin{array}{cc}6+4 \sqrt{2} & -2 \\ -2 & 6-4 \sqrt{2}\end{array}\right], \quad \zeta_{2} \otimes \zeta_{2}=\frac{1}{12}\left[\begin{array}{cc}6-4 \sqrt{2} & -2 \\ -2 & 6+4 \sqrt{2}\end{array}\right], \quad \zeta_{3} \otimes \zeta_{3}=\frac{1}{2}\left[\begin{array}{ll}1 & 1 \\ 1 & 1\end{array}\right]$, we validate that:

$$
\operatorname{det}\left(\frac{1}{12}\left[\begin{array}{ccc}
6+4 \sqrt{2} & 6-4 \sqrt{2} & 6 \\
-2 & -2 & 6 \\
6-4 \sqrt{2} & 6+4 \sqrt{2} & 6
\end{array}\right]\right) \neq 0
$$

Consequently, there exist linear mappings $\left\{\Psi_{k}: \mathbb{R}_{s y m}^{2 \times 2} \rightarrow \mathbb{R}\right\}_{k=1}^{3}$ yielding the unique decomposition:

$$
\forall G \in \mathbb{R}_{\text {sym }}^{2 \times 2} \quad G=\sum_{k=1}^{3} \Psi_{k}(G) \zeta_{k} \otimes \zeta_{k} .
$$

Now, since $\operatorname{Id}_{2}=\frac{3}{4} \zeta_{1} \otimes \zeta_{1}+\frac{3}{4} \zeta_{2} \otimes \zeta_{2}+\frac{1}{2} \zeta_{3} \otimes \zeta_{3}$, the continuity of each function $\Psi_{k}$ implies its positivity in a neighborhood of $\operatorname{Id}_{2}$ of some appropriate radius $r_{0}$.

2. For an arbitrary $G_{0} \in \mathbb{R}_{\text {sym, }}^{2 \times 2}$ we set:

$$
\forall k=1 \ldots 3 \quad \xi_{k}=\frac{1}{\left|G_{0}^{1 / 2} \zeta_{k}\right|} G_{0}^{1 / 2} \zeta_{k} \quad \text { and } \quad \Phi_{k}(G)=\left|G_{0}^{1 / 2} \zeta_{k}\right|^{2} \Psi_{k}\left(G_{0}^{-1 / 2} G G_{0}^{-1 / 2}\right) .
$$

Then, in view of (2.6) we obtain (2.5):

$$
\forall G \in \mathbb{R}_{\text {sym }}^{2 \times 2} \quad G=G_{0}^{-1 / 2}\left(\sum_{k=1}^{3} \Psi_{k}\left(G_{0}^{-1 / 2} G G_{0}^{-1 / 2}\right) \zeta_{k} \otimes \zeta_{k}\right) G_{0}^{1 / 2}=\sum_{k=1}^{3} \Phi_{k}(G) \xi_{k} \otimes \xi_{k},
$$


Finally, if $\left|G-G_{0}\right|<r\left(G_{0}\right)$ then $\left|G_{0}^{-1 / 2} G G_{0}^{-1 / 2}-\mathrm{Id}_{2}\right| \leq\left|G_{0}^{-1 / 2}\right|^{2}\left|G-G_{0}\right|<r_{0}$, and so indeed $\Phi_{k}(G)>0$, since $\Psi_{k}\left(G_{0}^{-1 / 2} G G_{0}^{-1 / 2}\right)>0$.

The above result can be localized in the following manner, similar to [42, Lemma 3.3]:

Lemma 2.4. There exists sequences of unit vectors $\left\{\eta_{k} \in \mathbb{R}^{2}\right\}_{k=1}^{\infty}$ and nonnegative smooth functions $\left\{\phi_{k} \in \mathcal{C}_{c}^{\infty}\left(\mathbb{R}_{\text {sym },>}^{2 \times 2}\right)\right\}_{k=1}^{\infty}$, such that:

$$
\forall G \in \mathbb{R}_{s y m,>}^{2 \times 2} \quad G=\sum_{k=1}^{\infty} \phi_{k}(G)^{2} \eta_{k} \otimes \eta_{k}
$$

and that:

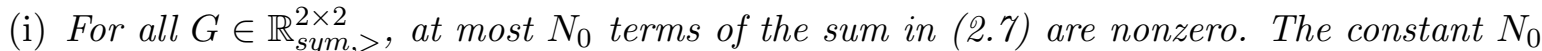
is independent of $G$.

(ii) For every compact $K \subset \mathbb{R}_{\text {sym, }}^{2 \times 2}$, there exists a finite set of indices $J(K) \subset \mathbb{N}$ such that $\phi_{k}(G)=0$ for all $k \notin J(K)$ and $G \in K$.

Proof. 1. Let $r_{0}$ be as in Lemma 2.3 and additionally ensure that:

$$
r_{0}<\frac{1}{8}
$$

Recall that for each $G \in \mathbb{R}_{\text {sym },>}^{2 \times 2}$ we have denoted $r(G)=\frac{r_{0}}{\left|G^{-1 / 2}\right|^{2}}$ and that $B(G, r(G)) \subset \mathbb{R}_{\text {sym },>}^{2 \times 2}$. We first construct a locally finite covering of $\mathbb{R}_{s y m,>}^{2 \times 2}$ with properties corresponding to (i) and (ii).

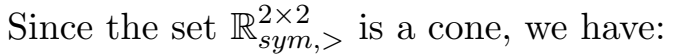

$$
\mathbb{R}_{\text {sym },>}^{2 \times 2}=\bigcup_{k \in \mathbb{Z}} 2^{k} \mathcal{C}_{0}, \quad \text { where } \quad \mathcal{C}_{0}=\left\{G \in \mathbb{R}_{\text {sym },>}^{2 \times 2} ; 1 / 2 \leq|G| \leq 1\right\} .
$$

The collection $\{B(G, r(G))\}_{G \in \mathcal{C}_{0}}$ covers the sector $\mathcal{C}_{0}$ by balls that have uniformly bounded radii: $r(G) \leq r_{0} \frac{|G|}{\sqrt{2}} \leq r_{0}$. Hence, by the Besicovitch covering theorem, it has a countable subcovering $\mathcal{G}_{0}=\bigcup_{\sigma=1}^{\sigma_{0}} \mathcal{G}_{0}^{\sigma}$, consisting of $\sigma_{0} \in \mathbb{N}$ countable families $\left\{\mathcal{G}_{0}^{\sigma}\right\}_{\sigma=1}^{\sigma_{0}}$ of pairwise disjoint balls.

Note that for all $c>0$ one has: $r(c G)=c r(G)$ and so: $B(c G, r(c G))=c B(G, r(G))$. Consequently, the collections $\mathcal{G}_{k}^{\sigma}=\left\{2^{k} B ; B \in \mathcal{G}_{0}^{\sigma}\right\}$ each consist of countably many pairwise disjoint balls, and $\mathcal{G}_{k}=\bigcup_{\sigma=1}^{\sigma_{0}} \mathcal{G}_{k}^{\sigma}$ is a covering of the dilated sector $2^{k} \mathcal{C}_{0}$, for every $k \in \mathbb{Z}$. Define:

$$
\forall \sigma=1 \ldots \sigma_{0} \quad \mathcal{G}_{\text {even }}^{\sigma}=\bigcup_{2 \mid k} \mathcal{G}_{k}^{\sigma} \quad \text { and } \quad \mathcal{G}_{\text {odd }}^{\sigma}=\bigcup_{2 \mid(k+1)} \mathcal{G}_{k}^{\sigma} .
$$

Clearly, in view of 2.9 , the $2 \sigma_{0}$ families in 2.10 form a covering of $\mathbb{R}_{s y m,>}^{2 \times 2}$, namely:

$$
\mathcal{G}=\bigcup_{\sigma=1}^{\sigma_{0}} \mathcal{G}_{\text {even }}^{\sigma} \cup \bigcup_{\sigma=1}^{\sigma_{0}} \mathcal{G}_{\text {odd }}^{\sigma} .
$$

We now prove that each of the families in $\mathcal{G}$ consists of pairwise disjoint balls. We argue by contradiction. Assume that:

$\exists G \in B\left(G_{1}, r\left(G_{1}\right)\right) \cap B\left(G_{2}, r\left(G_{2}\right)\right) \quad$ for some $\quad B\left(G_{1}, r\left(G_{1}\right)\right) \in \mathcal{G}_{2 k_{1}}^{\sigma}, \quad B\left(G_{2}, r\left(G_{2}\right)\right) \in \mathcal{G}_{2 k_{2}}^{\sigma}$.

Without loss of generality we may take $k_{1}=0$ and $k_{2}=k \geq 1$, so that:

$$
\frac{1}{2} \leq\left|G_{1}\right| \leq 1 \quad \text { and } \quad 2^{2 k-1} \leq\left|G_{2}\right| \leq 2^{2 k}
$$


This yields a contradiction with (2.8), in view of:

$$
\begin{aligned}
2^{2 k-1}-1 & \leq\left|G_{2}\right|-\left|G_{1}\right| \leq\left|G_{2}-G_{1}\right| \leq\left|G_{2}-G\right|+\left|G-G_{1}\right| \\
& \leq r\left(G_{2}\right)+r\left(G_{1}\right)=r_{0}\left(\frac{1}{\left|G_{2}^{-1 / 2}\right|^{2}}+\frac{1}{\left|G_{1}^{-1 / 2}\right|^{2}}\right) \leq \frac{r_{0}}{\sqrt{2}}\left(\left|G_{2}\right|+\left|G_{1}\right|\right) \leq r_{0}\left(2^{2 k}+1\right),
\end{aligned}
$$

2. Note that $\mathcal{G}$ can be assumed locally finite, by paracompactness. We write: $\mathcal{G}=\left\{B_{i}=\right.$ $\left.B\left(G_{i}, r\left(G_{i}\right)\right)\right\}_{i=1}^{\infty}$ and let $\left\{\theta_{i} \in \mathcal{C}_{c}^{\infty}\left(B_{i}\right)\right\}_{i=1}^{\infty}$ be a partition of unity subordinated to $\mathcal{G}$. For each $i \in \mathbb{N}$, let $\left\{\xi_{k, G_{i}}\right\}_{k=1}^{3}$ and $\left\{\Phi_{k, G_{i}}\right\}_{k=1}^{3}$ be the unit vectors and the linear functions as in Lemma 2.3. Then:

$$
\forall G \in \mathbb{R}_{\text {sym },>}^{2 \times 2} \quad G=\sum_{i \in \mathbb{N}} \theta_{i}(G) G=\sum_{i \in \mathbb{N}} \sum_{k=1}^{3} \theta_{i}(G) \Phi_{k, G_{i}}(G) \xi_{k, G_{i}} \otimes \xi_{k, G_{i}},
$$

and we see that 2.7) holds by taking:

$$
\eta_{i, k}=\xi_{k, G_{i}} \quad \text { and } \quad \phi_{i, k}=\left(\theta_{i} \Phi_{k, G_{i}}\right) .
$$

Since supp $\phi_{i, k} \subset \mathcal{B}_{i}$ and since each $G$ belongs to at most $2 \sigma_{0}$ balls $B_{i}$, we see that (i) holds with $N_{0}=6 \sigma_{0}$. On the other hand, condition (ii) follows by local finiteness of $\mathcal{G}$.

\section{The $\mathcal{C}^{1}$ Approximations - A Proof of Theorem 2.1.}

The first result in the approximating sequence construction is what corresponds to a 'step' in Nash and Kuiper's terminology.

Proposition 3.1. Let $\Omega \subset \mathbb{R}^{2}$ be an open and bounded set. Given are: functions $v \in \mathcal{C}^{\infty}(\bar{\Omega})$ and $w \in \mathcal{C}^{\infty}\left(\bar{\Omega}, \mathbb{R}^{2}\right)$, a nonnegative function $a \in \mathcal{C}^{\infty}(\bar{\Omega})$, and a unit vector $\eta \in \mathbb{R}^{2}$. Then, for every $\lambda>1$ there exist approximations $\tilde{v}_{\lambda} \in \mathcal{C}^{\infty}(\bar{\Omega})$ and $\tilde{w}_{\lambda} \in \mathcal{C}^{\infty}\left(\bar{\Omega}, \mathbb{R}^{2}\right)$ satisfying the following bounds:

$$
\begin{aligned}
\|\left(\frac{1}{2} \nabla \tilde{v}_{\lambda} \otimes \nabla \tilde{v}_{\lambda}+\operatorname{sym} \nabla \tilde{w}_{\lambda}\right)-\left(\frac{1}{2} \nabla v\right. & \left.\otimes \nabla v+\operatorname{sym} \nabla w+a^{2} \eta \otimes \eta\right) \|_{0} \\
& \leq \frac{C}{\lambda}\|a\|_{0}\left(\|\nabla a\|_{0}+\left\|\nabla^{2} v\right\|_{0}\right)+\frac{C}{\lambda^{2}}\|\nabla a\|_{0}^{2},
\end{aligned}
$$

$$
\begin{aligned}
& \forall x \in \bar{\Omega} \quad\left|\nabla \tilde{v}_{\lambda}(x)-\nabla v(x)\right| \leq C a(x)+\frac{C}{\lambda}\|\nabla a\|_{0}, \\
& \left|\nabla \tilde{w}_{\lambda}(x)-\nabla w(x)\right| \leq C a(x)\left(\|a\|_{0}+\|\nabla v\|_{0}\right)+\frac{C}{\lambda}\left(\|a\|_{0}\left(\|\nabla a\|_{0}+\left\|\nabla^{2} v\right\|_{0}\right)+\|\nabla a\|_{0}\|\nabla v\|_{0}\right) .
\end{aligned}
$$

Proof. Using the 1-periodic functions $\Gamma_{i}$ from Lemma 2.2, we define $\tilde{v}_{\lambda}$ and $\tilde{w}_{\lambda}$ as $\lambda$-periodic perturbations of $v, w$ in the direction $\eta$ :

$$
\begin{aligned}
& \tilde{v}_{\lambda}(x)=v(x)+\frac{1}{\lambda} \Gamma_{1}(x, \lambda x \cdot \eta) \\
& \tilde{w}_{\lambda}(x)=w(x)-\frac{1}{\lambda} \Gamma_{1}(x, \lambda x \cdot \eta) \nabla v(x)+\frac{1}{\lambda} \Gamma_{2}(x, \lambda x \cdot \eta) \eta .
\end{aligned}
$$


The error estimates in 3.2 follow immediately from (2.4). The pointwise error estimates (3.3) follow from $(2.4)$ in view of:

$$
\begin{aligned}
\nabla \tilde{v}_{\lambda}(x)=\nabla v(x)+ & \frac{1}{\lambda} \nabla_{x} \Gamma_{1}(x, \lambda x \cdot \eta)+\partial_{t} \Gamma_{1}(x, \lambda x \cdot \eta) \eta \\
\nabla \tilde{w}_{\lambda}(x)=\nabla w(x)- & \frac{1}{\lambda} \nabla v(x) \otimes \nabla_{x} \Gamma_{1}(x, \lambda x \cdot \eta)-\partial_{t} \Gamma_{1}(x, \lambda x \cdot \eta) \eta \otimes \nabla v(x)-\frac{1}{\lambda} \Gamma_{1}(x, \lambda x \cdot \eta) \nabla^{2} v(x) \\
& +\frac{1}{\lambda} \eta \otimes \nabla_{x} \Gamma_{2}(x, \lambda x \cdot \eta)+\partial_{t} \Gamma_{2}(x, \lambda x \cdot \eta) \eta \otimes \eta .
\end{aligned}
$$

Finally, we compute:

$$
\begin{aligned}
\frac{1}{2} \nabla \tilde{v}_{\lambda}(x) \otimes & \nabla \tilde{v}_{\lambda}(x)-\frac{1}{2} \nabla v(x) \otimes \nabla v(x) \\
= & \frac{1}{\lambda} \operatorname{sym}\left(\nabla v(x) \otimes \nabla_{x} \Gamma_{1}(x, \lambda x \cdot \eta)\right)+\partial_{t} \Gamma_{1}(x, \lambda x \cdot \eta) \operatorname{sym}(\nabla v(x) \otimes \eta) \\
& +\frac{1}{2}\left|\partial_{t} \Gamma_{1}(x, \lambda x \cdot \eta)\right|^{2} \eta \otimes \eta \\
& +\frac{1}{\lambda} \partial_{t} \Gamma_{1}(x, \lambda x \cdot \eta) \operatorname{sym}\left(\eta \otimes \nabla_{x} \Gamma_{1}(x, \lambda x \cdot \eta)\right)+\frac{1}{2 \lambda^{2}} \nabla_{x} \Gamma_{1}(x, \lambda x \cdot \eta) \otimes \nabla_{x} \Gamma_{1}(x, \lambda x \cdot \eta),
\end{aligned}
$$

and:

$$
\begin{aligned}
\operatorname{sym} \nabla \tilde{w}_{\lambda}(x) & -\operatorname{sym} \nabla w(x) \\
= & -\frac{1}{\lambda} \operatorname{sym}\left(\nabla v(x) \otimes \nabla_{x} \Gamma_{1}(x, \lambda x \cdot \eta)\right)-\partial_{t} \Gamma_{1}(x, \lambda x \cdot \eta) \operatorname{sym}(\nabla v(x) \otimes \eta) \\
& -\frac{1}{\lambda} \Gamma_{1}(x, \lambda x \cdot \eta) \nabla^{2} v(x)+\frac{1}{\lambda} \operatorname{sym}\left(\eta \otimes \nabla_{x} \Gamma_{2}(x, \lambda x \cdot \eta)\right) \\
& +\partial_{t} \Gamma_{2}(x, \lambda x \cdot \eta) \eta \otimes \eta
\end{aligned}
$$

We see that the terms in boxes cancel out, while the terms in double boxes add up to $a(x)^{2} \eta \otimes \eta$ in virtue of (2.3). Consequently:

$$
\begin{aligned}
&\left(\frac{1}{2} \nabla \tilde{v}_{\lambda}(x) \otimes \nabla \tilde{v}_{\lambda}(x)+\operatorname{sym} \nabla \tilde{w}_{\lambda}(x)\right)-\left(\frac{1}{2} \nabla v(x) \otimes \nabla v(x)+\operatorname{sym} \nabla w(x)+a(x)^{2} \eta \otimes \eta\right) \\
&= \frac{1}{\lambda}\left(\partial_{t} \Gamma_{1}(x, \lambda x \cdot \eta) \operatorname{sym}\left(\eta \otimes \nabla_{x} \Gamma_{1}(x, \lambda x \cdot \eta)\right)-\Gamma_{1}(x, \lambda x \cdot \eta) \nabla^{2} v(x)+\operatorname{sym}\left(\eta \otimes \nabla_{x} \Gamma_{2}(x, \lambda x \cdot \eta)\right)\right) \\
& \quad+\frac{1}{2 \lambda^{2}} \nabla_{x} \Gamma_{1}(x, \lambda x \cdot \eta) \otimes \nabla_{x} \Gamma_{1}(x, \lambda x \cdot \eta),
\end{aligned}
$$

which implies (3.1) in view of the bounds in 2.4).

We now complete the 'stage' in the approximating sequence construction.

Proposition 3.2. Let $\Omega \subset \mathbb{R}^{2}$ be an open and bounded domain. Let $v \in \mathcal{C}^{\infty}(\bar{\Omega}), w \in \mathcal{C}^{\infty}\left(\bar{\Omega}, \mathbb{R}^{2}\right)$ and $A \in \mathcal{C}^{\infty}\left(\bar{\Omega}, \mathbb{R}_{\text {sym }}^{2 \times 2}\right)$ be such that the deficit function $\mathcal{D}$ defined below is positive definite in $\bar{\Omega}$ :

$$
\exists c>0 \quad \mathcal{D}=A-\left(\frac{1}{2} \nabla v \otimes \nabla v+\operatorname{sym} \nabla w\right)>c \operatorname{Id}_{2} \quad \text { in } \bar{\Omega} .
$$


Fix $\varepsilon>0$. Then there exist $\tilde{v} \in \mathcal{C}^{\infty}(\bar{\Omega})$ and $\tilde{w} \in \mathcal{C}^{\infty}\left(\bar{\Omega}, \mathbb{R}^{2}\right)$ such that the new deficit $\tilde{\mathcal{D}}$ is still positive definite, and bounded by $\varepsilon$ together with the error in the approximations $\tilde{v}, \tilde{w}$, namely:

$$
\begin{gathered}
\exists \tilde{c}>0 \quad \tilde{\mathcal{D}}=A-\left(\frac{1}{2} \nabla \tilde{v} \otimes \nabla \tilde{v}+\operatorname{sym} \nabla \tilde{w}\right)>\tilde{c} \operatorname{Id}_{2} \quad \text { in } \bar{\Omega}, \\
\|\tilde{\mathcal{D}}\|_{0}<\varepsilon \quad \text { and } \quad\|\tilde{v}-v\|_{0}+\|\tilde{w}-w\|_{0}<\varepsilon .
\end{gathered}
$$

Moreover, we have the following uniform gradient error bounds:

$$
\|\nabla \tilde{v}-\nabla v\|_{0} \leq C N_{0}^{1 / 2}\|\mathcal{D}\|_{0}^{1 / 2} \quad \text { and } \quad\|\nabla \tilde{w}-\nabla w\|_{0} \leq C N_{0}\left(\|\nabla v\|_{0}+\|\mathcal{D}\|_{0}^{1 / 2}\right)\|\mathcal{D}\|_{0}^{1 / 2},
$$

where the constant $N_{0} \in \mathbb{N}$ is as in Lemma 2.4.

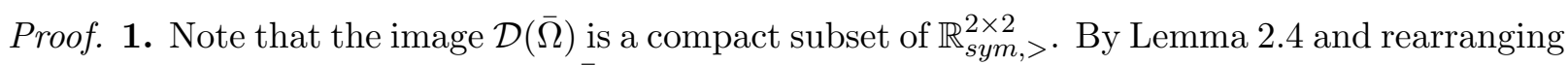
the indices, if needed, so that $J(\mathcal{D}(\bar{\Omega}))=\{1 \ldots N\}$ in (ii), we get:

$$
\forall x \in \bar{\Omega} \quad \mathcal{D}(x)=\sum_{k=1}^{N} b_{k}(x)^{2} \eta_{k} \otimes \eta_{k} \quad \text { where } \quad b_{k}=\phi_{k} \circ \mathcal{D} \in \mathcal{C}^{\infty}(\bar{\Omega}) .
$$

Let now $a_{k}=(1-\delta)^{1 / 2} b_{k}$, with $\delta>0$ so small that:

$$
\mathcal{D}-\sum_{k=1}^{N} a_{k}^{2} \eta_{k} \otimes \eta_{k}=\delta \mathcal{D} \quad \text { and } \quad \delta\|\mathcal{D}\|_{0}<\frac{\varepsilon}{2}
$$

We set $v_{1}=v, w_{1}=w$. For $k=1 \ldots N$ we inductively define $v_{k+1} \in \mathcal{C}^{\infty}(\bar{\Omega})$ and $w_{k+1} \in$ $\mathcal{C}^{\infty}\left(\bar{\Omega}, \mathbb{R}^{2}\right)$, by means of Proposition 3.1 applied to $v_{k}, w_{k}, a_{k}, \eta_{k}$ and with $\lambda_{k}>1$ sufficiently large as indicated below. We then finally set $\tilde{v}=v_{N+1}$ and $\tilde{w}=w_{N+1}$.

2. To prove the estimates $(3.6)-(3.8)$, we start by observing that since by Lemma 2.4 (i) at most $N_{0}$ terms in the expansion (3.9) are nonzero, there holds:

$$
\begin{aligned}
\sum_{k=1}^{N} a_{k}(x) \leq \sum_{k=1}^{N} b_{k}(x) & \leq N_{0}^{1 / 2}\left(\sum_{k=1}^{N} b_{k}(x)^{2}\right)^{1 / 2}=N_{0}^{1 / 2}(\text { Trace } \mathcal{D}(x))^{1 / 2} \\
& \leq N_{0}^{1 / 2}(\sqrt{2}|\mathcal{D}(x)|)^{1 / 2} \leq C N_{0}^{1 / 2}\|\mathcal{D}\|_{0}^{1 / 2} .
\end{aligned}
$$

Further, by 3.1$)$ and $(3.10)$ :

$$
\begin{aligned}
\tilde{\mathcal{D}} & =\mathcal{D}-\left(\left(\frac{1}{2} \nabla \tilde{v} \otimes \nabla \tilde{v}+\operatorname{sym} \nabla \tilde{w}\right)-\left(\frac{1}{2} \nabla v \otimes \nabla v+\operatorname{sym} \nabla w\right)\right) \\
= & \mathcal{D}-\sum_{k=1}^{N}\left(\left(\frac{1}{2} \nabla v_{k+1} \otimes \nabla v_{k+1}+\operatorname{sym} \nabla w_{k+1}\right)-\left(\frac{1}{2} \nabla v_{k} \otimes \nabla v_{k}+\operatorname{sym} \nabla w_{k}\right)\right) \\
= & \left(\mathcal{D}-\sum_{k=1}^{N} a_{k}^{2} \eta_{k} \otimes \eta_{k}\right) \\
& \quad-\sum_{k=1}^{N}\left(\left(\frac{1}{2} \nabla v_{k+1} \otimes \nabla v_{k+1}+\operatorname{sym} \nabla w_{k+1}\right)-\left(\frac{1}{2} \nabla v_{k} \otimes \nabla v_{k}+\operatorname{sym} \nabla w_{k}+a_{k}^{2} \eta_{k} \otimes \eta_{k}\right)\right) \\
= & \delta \mathcal{D}+\sum_{k=1}^{N} \mathcal{O}\left(\frac{1}{\lambda_{k}}\left(\left\|a_{k}\right\|_{0}\left\|\nabla a_{k}\right\|_{0}+\left\|\nabla a_{k}\right\|_{0}^{2}+\left\|a_{k}\right\|_{0}\left\|\nabla^{2} v_{k}\right\|_{0}\right)\right) .
\end{aligned}
$$


Choosing at each step $\lambda_{k}$ sufficiently large with respect to the given $a_{k}$ and the already generated $v_{k}$, we may ensure the smallness of the error term in the right hand side above and hence the positive definiteness of $\tilde{\mathcal{D}}$ in $(3.6)$, because of the uniform positive definiteness of: $\delta \mathcal{D}>c \delta \operatorname{Id}_{2}$ in $\bar{\Omega}$. Likewise, the first inequality in (3.7) follows already when the error is smaller than $\epsilon / 2$.

The same reasoning proves the error bounds on $\tilde{v}-v$ and $\tilde{w}-w$ in $(3.7)$, in view of $(3.2)$ :

$$
\begin{aligned}
\tilde{v}(x)-v(x) & =\sum_{k=1}^{N}\left(v_{k+1}(x)-v_{k}(x)\right)=\sum_{k=1}^{N} \mathcal{O}\left(\frac{1}{\lambda_{k}}\left\|a_{k}\right\|_{0}\right), \\
\tilde{w}(x)-w(x) & =\sum_{k=1}^{N}\left(w_{k+1}(x)-w_{k}(x)\right)=\sum_{k=1}^{N} \mathcal{O}\left(\frac{1}{\lambda_{k}}\left(\left\|a_{k}\right\|_{0}^{2}+\left\|\nabla a_{k}\right\|_{0}\left\|\nabla v_{k}\right\|_{0}\right)\right) .
\end{aligned}
$$

3. To obtain the first error bound in (3.8), use (3.3) and (3.11):

$$
|\nabla \tilde{v}(x)-\nabla v(x)| \leq \sum_{k=1}^{N}\left|\nabla v_{k+1}(x)-\nabla v_{k}(x)\right| \leq C \sum_{k=1}^{N} a_{k}(x)+\sum_{k=1}^{N} \mathcal{O}\left(\frac{1}{\lambda_{k}}\left\|a_{k}\right\|_{0}^{2}\right) \leq C N_{0}^{1 / 2}\|\mathcal{D}\|_{0}^{1 / 2},
$$

where again, by adjusting $\lambda_{k}$ at each step, we ensure the controllability of the error term with respect to the nonnegative quantity $N_{0}^{1 / 2}\|\mathcal{D}\|_{0}^{1 / 2}$. Likewise:

$$
\forall k=1 \ldots N \quad\left|\nabla v_{k}(x)\right| \leq|\nabla v(x)|+\sum_{i=1}^{k-1}\left|\nabla v_{i+1}(x)-\nabla v_{i}(x)\right| \leq\|\nabla v\|_{0}+C N_{0}^{1 / 2}\|\mathcal{D}\|_{0}^{1 / 2},
$$

and obviously by 3.11 :

which yield by (3.11):

$$
a_{k}(x) \leq \sum_{i=1}^{k-1} a_{i}(x) \leq C N_{0}^{1 / 2}\|\mathcal{D}\|_{0}^{1 / 2}
$$

$\sum_{k=1}^{N} a_{k}(x)\left(\left\|a_{k}\right\|_{0}+\left\|\nabla v_{k}\right\|_{0}\right) \leq C\left(\|\nabla v\|_{0}+N_{0}^{1 / 2}\|\mathcal{D}\|_{0}^{1 / 2}\right) \sum_{k=1}^{N} a_{k}(x) \leq C N_{0}\left(\|\nabla v\|_{0}+\|\mathcal{D}\|_{0}^{1 / 2}\right)\|\mathcal{D}\|_{0}^{1 / 2}$.

Consequently and by $(3.3)$, there follows the last gradient error bound in $(3.8)$ :

$$
\begin{aligned}
|\nabla \tilde{w}(x)-\nabla w(x)| \leq & \sum_{k=1}^{N}\left|\nabla w_{k+1}(x)-\nabla w_{k}(x)\right| \\
\leq & C \sum_{k=1}^{N} a_{k}(x)\left(\left\|a_{k}\right\|_{0}+\left\|\nabla v_{k}\right\|_{0}\right) \\
& \quad+\sum_{k=1}^{N} \mathcal{O}\left(\frac{1}{\lambda_{k}}\left(\left\|a_{k}\right\|_{0}\left\|\nabla a_{k}\right\|_{0}+\left\|a_{k}\right\|_{0}\left\|\nabla^{2} v_{k}\right\|_{0}+\left\|\nabla a_{k}\right\|_{0}\left\|\nabla v_{k}\right\|_{0}\right)\right) \\
\leq & C N_{0}\left(\|\nabla v\|_{0}+\|\mathcal{D}\|_{0}^{1 / 2}\right)\|\mathcal{D}\|_{0}^{1 / 2} .
\end{aligned}
$$

This concludes the proof of the stage approximation construction.

We now finally give:

Proof of Theorem 2.1. 
1. Fix $\varepsilon>0$. It suffices to construct $v \in \mathcal{C}^{1}(\bar{\Omega})$ and $w \in \mathcal{C}^{1}\left(\bar{\Omega}, \mathbb{R}^{2}\right)$ such that:

$$
A_{0}=\frac{1}{2} \nabla v \otimes \nabla v+\operatorname{sym} \nabla w \quad \text { in } \bar{\Omega}
$$

and:

$$
\left\|v-v_{0}\right\|_{0}+\left\|w-w_{0}\right\|_{0}<\varepsilon .
$$

The exact solution $(v, w)$ of $\left(3.12\right.$ will be obtained as the $\mathcal{C}^{1}$ limit of sequences of succesive approximations $\left\{v_{k} \in \mathcal{C}^{\infty}(\bar{\Omega}), w_{k} \in \mathcal{C}^{\infty}\left(\bar{\Omega}, \mathbb{R}^{2}\right)\right\}_{k=0}^{\infty}$, where $v_{0}$ and $w_{0}$ are given in the statement of the Theorem and satisfy (2.1), while $v_{k+1}$ and $w_{k+1}$ are defined inductively by means of Proposition 3.2 applied to $v_{k}, w_{k}$ and $\varepsilon_{k}>0$, under the following requirement:

$$
\sum_{k=1}^{\infty} \varepsilon_{k}<\varepsilon \quad \text { and } \quad \sum_{k=1}^{\infty} \varepsilon_{k}^{1 / 2}<1
$$

In agreement with our notation convention, we introduce the $k$-th deficit $\mathcal{D}_{k}$, which is positive definite by (3.6):

$$
\forall k \geq 0 \quad \mathcal{D}_{k}:=A_{0}-\left(\frac{1}{2} \nabla v_{k} \otimes \nabla v_{k}+\operatorname{sym} \nabla w_{k}\right) \in \mathcal{C}^{\infty}\left(\bar{\Omega}, \mathbb{R}_{\text {sym },>}^{2 \times 2}\right) .
$$

By (3.7) it follows that:

$$
\left\|v_{k}-v\right\|_{0}+\left\|w_{k}-w\right\|_{0} \leq \sum_{i=0}^{k-1}\left\|v_{i+1}-v_{i}\right\|_{0}+\sum_{i=0}^{k-1}\left\|w_{i+1}-w_{i}\right\|_{0}<\sum_{i=1}^{k-1} \epsilon_{i}<\sum_{i=1}^{\infty} \epsilon_{i} .
$$

Thus, $\left\{v_{k}\right\}_{k=0}^{\infty}$ and $\left\{w_{k}\right\}_{k=0}^{\infty}$ converge uniformly in $\bar{\Omega}$, respectively, to $v$ and $w$ which satisfy (3.13) in view of (3.14).

2. We now show that this convergence is in $\mathcal{C}^{1}$. Indeed, by 3.7$):\left\|\mathcal{D}_{k}\right\|_{0}<\epsilon_{k}$, so by 3.8 :

$$
\left\|\nabla v_{k+m}-\nabla v_{k}\right\|_{0} \leq \sum_{i=k}^{m-1}\left\|\nabla v_{i+1}-\nabla v_{i}\right\|_{0} \leq C N_{0}^{1 / 2} \sum_{i=k}^{m-1}\left\|\mathcal{D}_{i}\right\|_{0}^{1 / 2} \leq C N_{0}^{1 / 2} \sum_{i=k}^{m-1} \varepsilon_{i}^{1 / 2} .
$$

In particular, in view of (3.14) the sequence $\left\{\left\|\nabla v_{k}\right\|_{0}\right\}_{k=0}^{\infty}$ is bounded, so we further have:

$$
\begin{aligned}
\left\|\nabla w_{k+m}-\nabla w_{k}\right\|_{0} & \leq \sum_{i=k}^{m-1}\left\|\nabla w_{i+1}-\nabla w_{i}\right\|_{0} \\
& \leq C N_{0} \sum_{i=k}^{m-1}\left(\left\|\nabla v_{i}\right\|_{0}+\left\|\mathcal{D}_{i}\right\|_{0}^{1 / 2}\right)\left\|\mathcal{D}_{i}\right\|_{0}^{1 / 2} \leq \tilde{C} N_{0} \sum_{i=k}^{m-1} \varepsilon_{i}^{1 / 2},
\end{aligned}
$$

where the constant $\tilde{C}$ is independent of $k$ and $m$. Through the above assertions 3.15 and 3.16 , in view of the second condition in (3.14), we conclude that $\left\{v_{k}\right\}_{k=1}^{\infty}$ and $\left\{w_{k}\right\}_{k=0}^{\infty}$ are Cauchy sequences that converge in $\mathcal{C}^{1}(\bar{\Omega})$ to $v \in \mathcal{C}^{1}(\bar{\Omega})$ and $w \in \mathcal{C}^{1}\left(\bar{\Omega}, \mathbb{R}^{2}\right)$, respectively. Finally:

$$
\left\|A_{0}-\left(\frac{1}{2} \nabla v \otimes \nabla v+\operatorname{sym} \nabla w\right)\right\|_{0}=\lim _{k \rightarrow \infty}\left\|\mathcal{D}_{k}\right\|_{0} \leq \lim _{k \rightarrow \infty} \varepsilon_{k}=0
$$

implies 3.12 and completes the proof of Theorem 2.1. 
Remark 3.3. In addition to the uniform convergence postulated in Theorem 2.1 , one also has:

$$
\forall n \quad\left\|\nabla v_{n}\right\|_{0} \leq\left\|\nabla v_{0}\right\|_{0}+C N_{0}^{1 / 2}
$$

Using notation as in the proof above and recalling (3.15) and (3.14), this bound follows by:

$$
\left\|\nabla v-\nabla v_{0}\right\|_{0}=\lim _{k \rightarrow \infty}\left\|\nabla v_{k}-\nabla v_{0}\right\|_{0} \leq \lim _{k \rightarrow \infty}\left(C N_{0}^{1 / 2} \sum_{i=0}^{k-1} \epsilon_{i}^{1 / 2}\right) \leq C N_{0}^{1 / 2} .
$$

4. The $\mathcal{C}^{1, \alpha}$ approximations - a proof of Theorem 1.1, Preliminary Results and some HEURISTICS TOWARDS THE PROOF OF THEOREM 1.2 .

Theorem 1.1 follows easily from Theorem 1.2 , that will be proved in the next section.

\section{Proof of Theorem 1.1.}

Since $\mathcal{C}^{1}(\bar{\Omega})$ is dense in $\mathcal{C}^{0}(\bar{\Omega})$, we may without loss of generality assume that $v_{0} \in \mathcal{C}^{1}(\bar{\Omega})$. Set $w_{0}=0$ and $A_{0}=(\lambda+c) \operatorname{Id} \in \mathcal{C}^{0, \beta}\left(\bar{\Omega}, \mathbb{R}_{\text {sym }}^{2 \times 2}\right)$ where $c$ is a constant and $\lambda$ is constructed as follows.

Extend the function $f$ to $f \in L^{p}\left(\Omega_{\epsilon}\right)$ defined on an open smooth set $\Omega_{\epsilon} \supset \bar{\Omega}$ and solve:

$$
-\Delta \lambda=f \quad \text { in } \Omega_{\epsilon}, \quad \lambda=0 \quad \text { on } \partial \Omega_{\epsilon} .
$$

Since $\lambda \in W^{2, p}\left(\Omega_{\epsilon}\right)$, then Morrey's Theorem implies that $\lambda \in \mathcal{C}^{0, \beta}(\bar{\Omega})$ for every $\beta \in(0,1)$ when $p \geq 2$, and for $\beta=2-\frac{2}{p}$ when $p \in(1,2)$. Also, for $c$ large enough, condition 1.5 on the positive definiteness of the defect is satisfied. On the other hand:

$$
-\operatorname{curl} \operatorname{curl} A_{0}=-\Delta(\lambda+c)=f,
$$

so the result follows directly from Theorem 1.2 , since $\frac{2-2 / p}{2} \geq \frac{1}{7}$ is equivalent to $p \geq \frac{7}{6}$.

Our next simple Corollary concerns the steady-state Euler equations with the exchanged roles of the given pressure $q$ and the unknown forcing term $\nabla^{\perp} g$.

Corollary 4.1. Let $\Omega \subset \mathbb{R}^{2}$ be an open and bounded domain. Let $q \in \mathcal{C}^{0, \beta}(\bar{\Omega})$ for some $\beta \in(0,1)$ and fix $\epsilon>0$. Then for every exponent $\alpha$ in the range: $0<\alpha<\min \left\{\frac{1}{7}, \frac{\beta}{2}\right\}$, there exist sequences $\left\{u_{n} \in \mathcal{C}^{0, \alpha}\left(\bar{\Omega}, \mathbb{R}^{2}\right)\right\}_{n=1}^{\infty}$ and $\left\{g_{n} \in \mathcal{C}^{0, \alpha}(\bar{\Omega})\right\}_{n=1}^{\infty}$ solving in $\Omega$ the following system:

$$
\operatorname{div}\left(u_{n} \otimes u_{n}\right)-\nabla q=\nabla^{\perp} g_{n}, \quad \operatorname{div} u_{n}=0,
$$

and such that $u_{n}=\nabla^{\perp} v_{n}$ and $g_{n}=\operatorname{curl} w_{n}$, where each $v_{n} \in \mathcal{C}^{1, \alpha}(\bar{\Omega})$ and $w_{n} \in \mathcal{C}^{1}\left(\bar{\Omega}, \mathbb{R}^{2}\right)$, while the sequence $\left\{v_{n}\right\}_{n=1}^{\infty}$ is dense in $\mathcal{C}^{0}(\bar{\Omega})$ and $\left\|w_{n}\right\|_{0}<\epsilon$ for every $n \geq 1$.

Proof. As before, since $\mathcal{C}^{1}(\bar{\Omega})$ is dense in $\mathcal{C}^{0}(\bar{\Omega})$, it is enough to take $v_{0} \in \mathcal{C}^{1}(\bar{\Omega})$ and approximate it by a sequence $\left\{v_{n} \in \mathcal{C}^{1, \alpha}(\bar{\Omega})\right\}_{n=1}^{\infty}$ with the properties as in the statement of Corollary. Let $w_{0}=0$ and let $c>0$ be a sufficiently large constant, so that $(q+c) \operatorname{Id}_{2}-\nabla v_{0} \otimes \nabla v_{0}$ is strictly positive definite in $\bar{\Omega}$. By Theorem 1.2 , there exists sequences $v_{n} \in \mathcal{C}^{1, \alpha}(\bar{\Omega})$ and $w_{n} \in \mathcal{C}^{1, \alpha}\left(\bar{\Omega}, \mathbb{R}^{2}\right)$ which converge uniformly to $v_{0}$ and $w_{0}$ and which satisfy:

$$
(q+c) \operatorname{Id}_{2}=\nabla v_{n} \otimes \nabla v_{n}+2 \operatorname{sym} \nabla w_{n} \quad \text { in } \bar{\Omega} .
$$

Taking the cofactor of both sides in the above matrix identity, we get:

$$
(q+c) \operatorname{Id}_{2}=\nabla^{\perp} v_{n} \otimes \nabla^{\perp} v_{n}+2 \operatorname{cof}\left(\operatorname{sym} \nabla w_{n}\right) .
$$

Taking the row-wise divergence, we obtain 4.1 with $u_{n}=\nabla^{\perp} v_{n}$ and $g_{n}=\operatorname{curl} w_{n}$, since $\operatorname{div} \operatorname{cof} \nabla w_{n}=0$, while $\left(\operatorname{div} \operatorname{cof}\left(\nabla w_{n}\right)^{T}\right)^{\perp}=-\nabla\left(\operatorname{curl} w_{n}\right)$. 
Towards a proof of Theorem 1.2 we will derive a sequence of approximation results, and then combine them with Theorem 2.1 in section 6. For completeness, we first prove a simple, useful:

Lemma 4.2. Let $\Omega \subset \mathbb{R}^{2}$ be an open and bounded domain. Given are functions: $f \in \mathcal{C}^{N}\left(\bar{\Omega}, \mathbb{R}^{n}\right)$ and $\psi \in \mathcal{C}^{\infty}\left(\mathbb{R}^{n}, \mathbb{R}^{m}\right)$. Then:

$$
\forall k=0 \ldots N \quad\|\psi \circ f\|_{k} \leq M\|f\|_{k},
$$

where the constant $M>0$ depends on the dimensions $n, m$, the differentiability order $N$, the domain $\Omega$, the norm $\|\psi\|_{N}$ on the compact set $f(\bar{\Omega})$ and the norm $\|f\|_{0}$, but it does not depend on the higher norms of $f$.

Proof. The statement is obvious for $k=0$. Fix $k \in\{1 \ldots N\}$ and let $m=\left(m_{1}, \cdots, m_{k}\right)$ be any $k$-tuple of nonnegative integers such that $\sum_{i=1}^{k} i m_{i}=k$. Denoting $|m|=\sum_{i=1}^{k} m_{i}$ and using the interpolation inequality [1]:

$$
\forall i=1 \ldots k \quad\|f\|_{i} \leq M_{0}\|f\|_{0}^{1-i / k}\|f\|_{k}^{i / k}
$$

valid with a constant $M_{0}>0$ depending on $n, N$ and $\Omega$, we get:

$$
\prod_{i=1}^{k}\left\|\nabla^{i} f\right\|_{0}^{m_{i}} \leq M_{0}^{|m|} \prod_{i=1}^{k}\|f\|_{0}^{m_{i}-i m_{i} / k}\|f\|_{k}^{i m_{i} / k}=M_{0}^{|m|}\|f\|_{0}^{|m|-1}\|f\|_{k} .
$$

with $|m|:=m_{1}+\cdots+m_{j}$. Calculating the partial derivatives in $\nabla^{k}(\psi \circ f)$ by the Faà di Bruno formula, gives hence the desired estimate:

$$
\left\|\nabla^{k}(\psi \circ f)\right\|_{0} \leq M \sum_{m} \prod_{i=1}^{k}\left\|\nabla^{i} f\right\|_{0}^{m_{i}} \leq M\|f\|_{k} .
$$

Above, the summation extends over all multiindices $m=\left(m_{1}, \cdots, m_{k}\right)$ with the properties listed at the beginning of the proof.

We recall the following estimates which have been proved in [10]:

Lemma 4.3. Let $\varphi \in \mathcal{C}_{c}^{\infty}(B(0,1), \mathbb{R})$ be a standard mollifier supported on the ball $B(0,1) \subset \mathbb{R}^{n}$, that is a nonnegative, smooth and radially symmetric function such that $\int_{\mathbb{R}^{n}} \varphi=1$. Denote:

$$
\forall l \in(0,1) \quad \varphi_{l}(x)=\frac{1}{l^{n}} \varphi\left(\frac{x}{l}\right) .
$$

Then, for every $f, g \in \mathcal{C}^{0}\left(\mathbb{R}^{n}\right)$ there holds:

$$
\begin{aligned}
\forall k, j \geq 0 & \left\|f * \varphi_{l}\right\|_{k+j} \leq \frac{C}{l^{k}}\|f\|_{j} \\
\forall k \geq 0 & \left\|f * \varphi_{l}-f\right\|_{k} \leq \frac{C}{l^{k-2}}\|f\|_{2} \\
\forall \alpha \in(0,1] & \left\|f * \varphi_{l}-f\right\|_{0} \leq C l^{\alpha}\|f\|_{0, \alpha} \\
\forall \alpha \in(0,1] & \left\|f * \varphi_{l}\right\|_{1} \leq \frac{C}{l^{1-\alpha}}\|f\|_{0, \alpha} \\
\forall k \geq 0 \quad \forall \alpha \in(0,1] & \left\|(f g) * \varphi_{l}-\left(f * \varphi_{l}\right)\left(g * \varphi_{l}\right)\right\|_{k} \leq \frac{C}{l^{k-2 \alpha}}\|f\|_{0, \alpha}\|g\|_{0, \alpha},
\end{aligned}
$$

with the uniform constants $C>0$ depending only on the smoothness exponents $k, j, \alpha$. 
Proof. The estimate (4.2) follows directly from the definition of convolution. To prove (4.3), note that for every $x \in \mathbb{R}^{n}$ :

$$
\begin{aligned}
& \left|\nabla^{k}\left(f * \varphi_{l}-f\right)(x)\right|=\left|\int_{\mathbb{R}^{n}} \varphi_{l}(y)\left(\nabla^{k} f(x-y)-\nabla^{k} f(x)\right) \mathrm{d} y\right| \\
& \quad=\left|\int_{\mathbb{R}^{n}} \nabla^{k} \varphi_{l}(y)(f(x-y)-f(x)) \mathrm{d} y\right|=\frac{1}{l^{k}}\left|\int_{\mathbb{R}^{n}} \frac{1}{l^{n}} \nabla^{k} \varphi\left(\frac{y}{l}\right)\left(\nabla f(x) \cdot y+r_{x}(y)\right) \mathrm{d} y\right| \\
& \quad=\frac{1}{l^{k}}\left|\int_{\mathbb{R}^{n}} \frac{1}{l^{n}} \nabla^{k} \varphi\left(\frac{y}{l}\right) r_{x}(y) \mathrm{d} y\right| \leq \frac{C}{l^{k}} \sup _{x \in \mathbb{R}^{n} ;|y|<l}\left|r_{x}(y)\right| \leq \frac{C}{l^{k-2}}\|f\|_{2},
\end{aligned}
$$

where we integrated by parts, discarded the contribution with the symmetric term $\nabla f(x) \cdot y$ which integrates to 0, and estimated the Taylor's formula remainder term:

$$
r_{x}(y)=f(x-y)-f(x)-\nabla f(x) \cdot y=\|f\|_{2} \mathcal{O}\left(|y|^{2}\right) .
$$

The proof of (4.4) follows similarly by:

$$
\begin{aligned}
\left|\nabla^{k}\left(f * \varphi_{l}-f\right)(x)\right| & =\left.\left|\int_{\mathbb{R}^{n}} \varphi_{l}(y)\right| y\right|^{\alpha} \frac{f(x-y)-f(x)}{|y|^{\alpha}} \mathrm{d} y \mid \\
& \leq C l^{\alpha}\|f\|_{0, \alpha} \int_{\mathbb{R}^{n}} \varphi_{l}(y) \mathrm{d} y \leq C l^{\alpha}\|f\|_{0, \alpha},
\end{aligned}
$$

while for (4.5) we write:

$$
\begin{aligned}
\left|\nabla\left(f * \varphi_{l}\right)(x)\right| & =\left|\int_{\mathbb{R}^{n}} f(x-y) \frac{1}{l^{n+1}} \nabla \varphi_{l}\left(\frac{y}{l}\right) \mathrm{d} y\right|=\frac{1}{l}\left|\int_{\mathbb{R}^{n}} \frac{f(x-y)-f(x)}{|y|^{\alpha}} \frac{|y|^{\alpha}}{l} \frac{1}{l^{n}} \nabla \varphi_{l}\left(\frac{y}{l}\right) \mathrm{d} y\right| \\
& \leq C l^{\alpha-1}\|f\|_{0, \alpha} \int_{\mathbb{R}^{n}} \frac{1}{l^{n}}\left|\nabla \varphi_{l}\left(\frac{y}{l}\right)\right| \mathrm{d} y \leq \frac{C}{l^{1-\alpha}}\|f\|_{0, \alpha} .
\end{aligned}
$$

Finally, for the crucial commutator estimate (4.6) we refer to [10, Lemma 1].

\section{A heuristic overview of the next two sections.}

Let us attempt to follow the construction in sections 2 and 3 , but with the goal of controlling the higher Hölder norms of the iterations, and hence also quantifying the growth of the $\mathcal{C}^{2}$ norms of $v, w$. Let $A \in \mathcal{C}^{\infty}\left(\bar{\Omega}, \mathbb{R}_{\text {sym }}^{2 \times 2}\right)$ be the target matrix field and let $v_{1} \in \mathcal{C}^{\infty}(\bar{\Omega}), w_{1} \in \mathcal{C}^{\infty}\left(\bar{\Omega}, \mathbb{R}^{2}\right)$ be given at an input of a 'stage'. As in Proposition 3.2 , we decompose the defect $\mathcal{D}=A-\left(\frac{1}{2} \nabla v_{1} \otimes\right.$ $\left.\nabla v_{1}+\operatorname{sym} \nabla w_{1}\right)$ into a linear combination $\sum_{k=1}^{N} a_{k}^{2} \eta_{k} \otimes \eta_{k}$ of rank-one symmetric matrices with smooth coefficients given by Lemma 2.4. We define:

$$
\begin{aligned}
v_{k+1}(x) & =v_{k}(x)+\frac{1}{\lambda} \Gamma_{1}\left(x, \lambda x \cdot \eta_{k}\right), \\
w_{k+1}(x) & =w_{k}(x)-\frac{1}{\lambda} \Gamma_{1}\left(x, \lambda x \cdot \eta_{k}\right) \nabla v_{k}(x)+\frac{1}{\lambda} \Gamma_{2}\left(x, \lambda x \cdot \eta_{k}\right) \eta_{k} .
\end{aligned}
$$


This yields, by applying Lemma 4.2 to $\psi(x)=x^{2}$ and $f=a_{k}$ :

$$
\begin{aligned}
& \forall m: 0 \ldots 3 \quad\left\|\nabla^{m} v_{k+1}-\nabla^{m} v_{k}\right\|_{0} \leq C \sum_{i+j=m ; 0 \leq i, j \leq m}\left\|a_{k}\right\|_{i} \lambda^{j-1}, \\
& \forall m: 0 \ldots 2 \quad\left\|\nabla^{m} w_{k+1}-\nabla^{m} w_{k}\right\|_{0} \leq C \sum_{i+j=m ; 0 \leq i, j \leq m}\left\|a_{k}\right\|_{i} \lambda^{j-1} \\
& \quad+C \sum_{i+j+s=m ; 0 \leq i, j, s \leq m}\left\|a_{k}\right\|_{i} \lambda^{j-1}\left\|\nabla^{s+1} v_{k}\right\|_{0},
\end{aligned}
$$

On the other hand, applying Lemma 4.2 to $\psi=\phi_{k}$ defined in Lemma 2.4 and to $f=\mathcal{D}$, we get:

$$
\forall k: 1 \ldots N \quad\left\|a_{k}\right\|_{2} \leq C\left(\left\|v_{1}\right\|_{3}^{2}+\left\|w_{1}\right\|_{3}+\|A\|_{2}\right) .
$$

Now, in order to control the $\mathcal{C}^{1, \alpha}$ norm of $v_{N+1}$ through interpolation, we need to control the norm $\left\|v_{N+1}\right\|_{2}$, which in turn depends on $\left\|a_{k}\right\|_{2}$. The above estimate shows that at the end of each stage, the $\mathcal{C}^{2}$ norm of $a_{k}$ is determined by the $\mathcal{C}^{3}$ norms of the given $v_{1}$ and $w_{1}$ of the previous stage. Further, the $\mathcal{C}^{2}$ norm of $w_{N+1}$ is only controlled by the $\mathcal{C}^{3}$ norm of $v_{0}$ and also of all the $a_{k}$ 's. One might hope to control $\left\|a_{k}\right\|_{3}$ if the deficit $\mathcal{D}$ is small enough, but the dependence of $\left\|w_{N+1}\right\|_{2}$ on $\left\|v_{0}\right\|_{3}$ cannot be easily bypassed. Recalling that we need infinitely many stages in the construction, this implies that a direct estimate cannot be obtained in this manner, unless we deal with analytic data similar as in [4]. We thus need to modify the previous simplistic approach.

The appropriate modification is achieved by introducing a mollification before each stage. Indeed, we note that the loss of derivatives in the above estimates is accompanied by a similar gain in the powers of $\lambda$, in a manner that the total order of derivatives, plus the order of powers needed to control $\left\|v_{N+1}\right\|_{2}$ and $\left\|w_{N+1}\right\|_{2}$ is constant. If we replace $v_{1}$ and $w_{1}$ by their mollifications on the scale $l \sim \lambda^{-1}$, each derivative loss can be estimated by one power of $\lambda$, and $\left\|v_{0}\right\|_{2}$ and $\left\|w_{0}\right\|_{2}$ will control $\left\|v_{N+1}\right\|_{2}$ and $\left\|w_{N+1}\right\|_{2}$. One problem still remains to be taken care of: does the deficit $\mathcal{D}$ decrease at the end of each stage? As the calculation below will show, a mollification of order $\lambda^{-1}$ does not suffice to this end, and we need to mollify at a larger scale of $l>\lambda^{-1}$.

This is indeed how we want proceed. In practice, we let the mollification scale to be $l=\delta / M$ and we treat $\nabla v$ "like $a$ ", controlling its $j$-th norm by $\delta l^{-j}$. We then "sacrifice" one $l$ in order to gain one $\delta$; instead of $\left\|\nabla\left(v * \varphi_{l}\right)\right\|_{j} \leq C\|v\|_{1} l^{-j}$, we use $\left\|\nabla\left(v * \varphi_{l}\right)\right\|_{j} \leq C\left(\|v\|_{2} l\right) l^{-j}$, choosing $l$ such that $l\|v\|_{2}<\delta$ and obtaining the desired bound (5.2).

Finally, note that the loss of $N$ powers of $\lambda l>1$ in the control of the $\mathcal{C}^{2}$ norms at the end of each stage, is the main reason why the described scheme does not deliver better than $\mathcal{C}^{1,1 / 7}$ estimates, even for the optimal $N=3$ from the decomposition in Lemma 2.3 .

\section{The $\mathcal{C}^{1, \alpha}$ Approximations - A 'SteP' And A 'Stage' in A Proof of Theorem 1.2 ,}

In this section, we develop the approximation technique that will be used for a proof of Theorem 1.2 in the next section. The first result is a variant of Proposition 3.1 in which we accomplish the 'step' of the Nash-Kuiper construction with extra estimates on the higher derivatives.

Proposition 5.1. Let $\Omega \subset \mathbb{R}^{2}$ be an open, bounded set. Given are functions: $v \in \mathcal{C}^{3}(\bar{\Omega}), w \in$ $\mathcal{C}^{2}\left(\bar{\Omega}, \mathbb{R}^{2}\right)$, a nonnegative function $a \in \mathcal{C}^{3}(\bar{\Omega})$ and a unit vector $\eta \in \mathbb{R}^{2}$. Let $\delta, l \in(0,1)$ be two parameter constants such that:

$$
\|a\|_{m} \leq \frac{\delta}{l^{m}} \quad \forall m=0 \ldots 3, \quad \text { and } \quad\|\nabla v\|_{m} \leq \frac{\delta}{l^{m}} \quad \forall m=1,2 .
$$


Then for every $\lambda>1 / l$ there exist approximating functions $\tilde{v}_{\lambda} \in \mathcal{C}^{3}(\bar{\Omega})$ and $\tilde{w}_{\lambda} \in \mathcal{C}^{2}\left(\bar{\Omega}, \mathbb{R}^{2}\right)$ satisfying the following bounds, with a universal constant $C>0$ independent of all parameters:

$$
\begin{gathered}
\left\|\left(\frac{1}{2} \nabla \tilde{v}_{\lambda} \otimes \nabla \tilde{v}_{\lambda}+\operatorname{sym} \nabla \tilde{w}_{\lambda}\right)-\left(\frac{1}{2} \nabla v \otimes \nabla v+\operatorname{sym} \nabla w+a^{2} \eta \otimes \eta\right)\right\|_{0} \leq C \frac{\delta^{2}}{\lambda l}, \\
\left\|\tilde{v}_{\lambda}-v\right\|_{m} \leq C \delta \lambda^{m-1} \quad \forall m=0 \ldots 3, \\
\left\|\tilde{w}_{\lambda}-w\right\|_{m} \leq C \delta \lambda^{m-1}\left(1+\|\nabla v\|_{0}\right) \quad \forall m=0 \ldots 2 .
\end{gathered}
$$

Proof. We define $\tilde{v}_{\lambda}, \tilde{w}_{\lambda}$ as in the proof of Proposition 3.1 .

$$
\begin{aligned}
\tilde{v}_{\lambda}(x) & =v(x)+\frac{1}{\lambda} \Gamma_{1}(x, \lambda x \cdot \eta), \\
\tilde{w}_{\lambda}(x) & =w(x)-\frac{1}{\lambda} \Gamma_{1}(x, \lambda x \cdot \eta) \nabla v(x)+\frac{1}{\lambda} \Gamma_{2}(x, \lambda x \cdot \eta) \eta .
\end{aligned}
$$

Firstly, (5.2) follows immediately from (3.1) in view of (5.1), because $\lambda l>1$ :

$$
\frac{1}{\lambda}\|a\|_{0}\left(\|\nabla a\|_{0}+\left\|\nabla^{2} v\right\|_{0}\right)+\frac{1}{\lambda^{2}}\|\nabla a\|_{0}^{2} \leq 2 \frac{\delta}{\lambda} \frac{\delta}{l}+\frac{1}{\lambda^{2}} \frac{\delta^{2}}{l^{2}} \leq 3 \frac{\delta^{2}}{\lambda l} .
$$

To check 5.3), we compute directly as in Lemma 2.2 .

$$
\nabla^{m}\left(\tilde{v}_{\lambda}-v\right)\left\|_{0} \leq \frac{C}{\lambda}\right\| \nabla^{m} \Gamma_{1}(x, \lambda x \cdot \eta)\left\|_{0} \leq \frac{C}{\lambda} \sum_{i+j=m ; 0 \leq i, j \leq m}\right\| a \|_{j} \lambda^{j} \leq \frac{C}{\lambda} \sum_{i=0}^{m} \frac{\delta}{l^{i}} \lambda^{m-i} \leq C \delta \lambda^{m-1}
$$

by (5.1) and noting again $\lambda l>1$. Similarly:

$$
\begin{aligned}
\left\|\nabla^{m}\left(\tilde{w}_{\lambda}-w\right)\right\|_{0} & \leq \frac{C}{\lambda}\left(\left\|\nabla^{m} \Gamma_{2}(x, \lambda x \cdot \eta)\right\|_{0}+\left\|\nabla^{m} \Gamma_{1}(x, \lambda x \cdot \eta) \nabla v\right\|_{0}\right) \\
& \leq \frac{C}{\lambda}\left(\sum_{i+j=m, 0 \leq i, j \leq m}\left\|a^{2}\right\|_{i} \lambda^{j}+\sum_{i+j+s=m, 0 \leq i, j, s \leq m}\|a\|_{i} \lambda^{j}\|\nabla v\|_{s}\right) \\
& \leq \frac{C}{\lambda}\left(\sum_{i=1}^{m} \frac{\delta}{l^{i}} \lambda^{m-i}+\sum_{0 \leq i+s \leq m, 0 \leq i, s \leq m} \frac{\delta}{l^{i}} \lambda^{m-(i+s)} \frac{\delta}{l^{s}}+\sum_{i+j=m, 0 \leq i, j \leq m} \frac{\delta}{l^{i}} \lambda^{j}\|\nabla v\|_{0}\right) \\
& \leq \frac{C}{\lambda}\left(\sum_{i=1}^{m} \frac{\delta}{l^{i}} \lambda^{m-i}\right)\left(1+1+\|\nabla v\|_{0}\right) \leq C \delta \lambda^{m-1}\left(1+\|\nabla v\|_{0}\right),
\end{aligned}
$$

where we applied Lemma 4.2 to $\psi(x)=x^{2}$ and $f=a$ in view of (5.1) yielding $\|a\|_{0} \leq 1$, so that: $\left\|a^{2}\right\|_{i} \leq C\|a\|_{i} \leq C \delta / l^{i}$. This achieves (5.4) and completes the proof of Proposition.

We now accomplish the 'stage' in the Hölder regular approximation construction.

Proposition 5.2. Let $\Omega \subset \mathbb{R}^{2}$ be an open, bounded domain. Let $v \in \mathcal{C}^{2}(\bar{\Omega}), w \in \mathcal{C}^{2}\left(\bar{\Omega}, \mathbb{R}^{2}\right)$ and $A \in \mathcal{C}^{0, \beta}\left(\bar{\Omega}, \mathbb{R}_{\text {sym }}^{2 \times 2}\right)$ for some $\beta \in(0,1)$, be such that the deficit $\mathcal{D}$ is appropriately small:

$$
\mathcal{D}=A-\left(\frac{1}{2} \nabla v \otimes \nabla v+\operatorname{sym} \nabla w\right), \quad 0<\|\mathcal{D}\|_{0}<\delta_{0} \ll 1 .
$$

Then, for every two parameter constants $M, \sigma$ satisfying:

$$
M>\max \left\{\|v\|_{2},\|w\|_{2}, 1\right\} \quad \text { and } \quad \sigma>1,
$$


there exists $\tilde{v} \in \mathcal{C}^{2}(\bar{\Omega})$ and $\tilde{w} \in \mathcal{C}^{2}\left(\bar{\Omega}, \mathbb{R}^{2}\right)$ such that the following error bounds hold for $\tilde{v}, \tilde{w}$ and the new deficit $\tilde{\mathcal{D}}=A-\left(\frac{1}{2} \nabla \tilde{v} \otimes \nabla \tilde{v}+\operatorname{sym} \nabla \tilde{w}\right)$ :

$$
\begin{gathered}
\|\tilde{\mathcal{D}}\|_{0} \leq C\left(\frac{\|A\|_{0, \beta}}{M^{\beta}}\|\mathcal{D}\|_{0}^{\beta / 2}+\frac{1}{\sigma}\|\mathcal{D}\|_{0}\right), \\
\|\tilde{v}-v\|_{1} \leq C\|\mathcal{D}\|_{0}^{1 / 2} \quad \text { and } \quad\|\tilde{w}-w\|_{1} \leq C\left(1+\|\nabla v\|_{0}\right)\|\mathcal{D}\|_{0}^{1 / 2}, \\
\|\tilde{v}\|_{2} \leq C M \sigma^{3} \quad \text { and } \quad\|\tilde{w}\|_{2} \leq C\left(1+\|\nabla v\|_{0}\right) M \sigma^{3} .
\end{gathered}
$$

The constant $C>0$ is universal and independent of all parameters.

Proof. Analogously to [10, Proposition 4], the proof is split into three parts.

1. Mollification. Let $\varphi \in \mathcal{C}_{c}^{\infty}(B(0,1))$ be the standard mollifier in $2 \mathrm{~d}$, as in Lemma 4.3. Since $v, w$ and $A$ can be extended on the whole $\mathbb{R}^{2}$, with all their relevant norms increased at most $C$ times $(C$ depends here on the curvature of the boundary $\partial \Omega$ ), we may define:

$$
\mathfrak{v}=v * \varphi_{l}, \quad \mathfrak{w}:=w * \varphi_{l}, \quad \mathfrak{A}:=A * \varphi_{l} \quad \text { with } \quad l=\frac{\|\mathcal{D}\|_{0}^{1 / 2}}{M}<1 .
$$

Applying Lemma 4.3 and noting (5.6), we immediately get the following uniform error bounds for $\mathfrak{v}, \mathfrak{w}, \mathfrak{A}$ and for the induced deficit $\mathfrak{D}=\mathfrak{A}-\left(\frac{1}{2} \nabla \mathfrak{v} \otimes \nabla \mathfrak{v}+\operatorname{sym} \nabla \mathfrak{w}\right)$ :

$$
\begin{aligned}
\|\mathfrak{v}-v\|_{1}+\|\mathfrak{w}-w\|_{1} & \leq C l\left(\|v\|_{2}+\|w\|_{2}\right) \leq C\|\mathcal{D}\|_{0}^{1 / 2}, \\
\|\mathfrak{A}-A\|_{0} & \leq C l^{\beta}\|A\|_{0, \beta}, \\
\|\mathfrak{D}\|_{m} & \leq\left\|\mathcal{D} * \varphi_{l}\right\|_{m}+\left\|\left(\nabla v * \varphi_{l}\right) \otimes\left(\nabla v * \varphi_{l}\right)-(\nabla v \otimes \nabla v) * \varphi_{l}\right\|_{m} \\
& \leq \frac{C}{l^{m}}\|\mathcal{D}\|+\frac{C}{l^{m-2}}\|v\|_{2}^{2} \leq \frac{C}{l^{m}}\|\mathcal{D}\|_{0} \quad \forall m=0 \ldots 3 .
\end{aligned}
$$

In the proof of the last inequality above, we used (4.6) with the Hölder exponent $\alpha=1$.

We note that so far we have simply exchanged the lower regularity fields $v, w, A$ with their smooth approximations, at the expense of the error that, as we shall see below, is compatible with the that postulated in (5.7) - 5.9). The following estimate, however, reflects the advantage of averaging through mollification that results in the control of $\mathcal{C}^{3}$ norm of $\mathfrak{v}$ by the $\mathcal{C}^{2}$ norm:

$$
\forall m=1,2 \quad\|\nabla \mathfrak{v}\|_{m} \leq\|\mathfrak{v}\|_{m+1} \leq \frac{C}{l^{m-1}}\|v\|_{2} \leq \frac{C}{l^{m-1}}\|\mathcal{D}\|_{0}^{1 / 2},
$$

where again we used Lemma 4.3 and (5.6). Note that the scaling bound (5.11) is consistent with the second requirement in (5.1) of Proposition 5.1. We also record the following simple bound:

$$
\|\mathfrak{w}\|_{2} \leq C\|w\|_{2} \leq C M \text {. }
$$

2. Modification and positive definiteness. Contrary to the 'stage' construction in the proof of Proposition 3.2 , we do not know whether the original defect $\mathcal{D}$ (and hence the induced defect $\mathfrak{D}$ ) is positive definite, so that Lemma 2.4 could be used. In any case, we need to keep the number of terms in the decomposition (3.9) into rank-one matrices as small as possible.

We now further modify $\mathfrak{w}$ in order to use the optimal decomposition in (2.5). Let $r_{0}$ be as in Lemma 2.3 and define:

$$
\mathfrak{w}^{\prime}=\mathfrak{w}-2 \frac{\left(\|\mathfrak{D}\|_{0}+\|\mathcal{D}\|_{0}\right)}{r_{0}} i d_{2}, \quad \mathfrak{D}^{\prime}=\mathfrak{A}-\left(\frac{1}{2} \nabla \mathfrak{v} \otimes \nabla \mathfrak{v}+\operatorname{sym} \nabla \mathfrak{w}^{\prime}\right) .
$$


Clearly, by 5.10 we get:

$$
\left\|\mathfrak{w}^{\prime}-\mathfrak{w}\right\|_{2} \leq C\left(\|\mathfrak{D}\|_{0}+\|\mathcal{D}\|_{0}\right) \leq C\|\mathcal{D}\|_{0}
$$

Note now that:

$$
\mathfrak{D}^{\prime}(x)=2 \frac{\left(\|\mathfrak{D}\|_{0}+\|\mathcal{D}\|_{0}\right)}{r_{0}} \operatorname{Id}_{2}+\mathfrak{D}(x)=2 \frac{\left(\|\mathfrak{D}\|_{0}+\|\mathcal{D}\|_{0}\right)}{r_{0}}\left(\operatorname{Id}_{2}+\frac{r_{0}}{2\left(\|\mathfrak{D}\|_{0}+\|\mathcal{D}\|_{0}\right)} \mathfrak{D}\right) \quad \forall x \in \bar{\Omega} .
$$

By Lemma 2.3 we may apply 2.5 to the scaled defect $G=\operatorname{Id}_{2}+\frac{r_{0}}{2\left(\|\mathfrak{D}\|_{0}+\|\mathcal{D}\|_{0}\right)} \mathfrak{D}$ and arrive at:

$$
\mathfrak{D}^{\prime}(x)=\sum_{k=1}^{3} 2 \frac{\left(\|\mathfrak{D}\|_{0}+\|\mathcal{D}\|_{0}\right)}{r_{0}} \Phi_{k}(G(x)) \xi_{k} \otimes \xi_{k}=\sum_{k=1}^{3} a_{k}^{2}(x) \xi_{k} \otimes \xi_{k} \quad \forall x \in \bar{\Omega},
$$

where $\left\{a_{k}=\left(2 \frac{\left(\|\mathfrak{D}\|_{0}+\|\mathcal{D}\|_{0}\right)}{r_{0}} \Phi_{k} \circ G\right)^{1 / 2}\right\}_{k=1}^{3}$ are positive smooth functions on $\bar{\Omega}$. We claim that:

$$
\forall k=1 \ldots 3 \quad \forall m=0 \ldots 3 \quad\left\|a_{k}\right\|_{m} \leq \frac{C}{l^{m}}\|\mathcal{D}\|_{0}^{1 / 2} .
$$

Indeed, for $m=0$ this inequality follows directly by $\|\mathfrak{D}\|_{0} \leq C\|\mathcal{D}\|_{0}$. For $m=1 \ldots 3$ we use Lemma 4.2 to each $\psi=\Phi_{k}^{1 / 2}$ and $f=G$, where noting that $\|G\|_{0} \leq C$ and recalling 5.10 yields:

$$
\begin{aligned}
\left\|a_{k}\right\|_{m} & \leq\left(2 \frac{\left(\|\mathfrak{D}\|_{0}+\|\mathcal{D}\|_{0}\right)}{r_{0}}\right)^{1 / 2} C\|G\|_{m} \\
& \leq C\left(\|\mathfrak{D}\|_{0}+\|\mathcal{D}\|_{0}\right)^{1 / 2}\left(C+\frac{r_{0}}{2\left(\|\mathfrak{D}\|_{0}+\|\mathcal{D}\|_{0}\right)}\|\mathfrak{D}\|_{m}\right) \\
& \leq C\left(\left(\|\mathfrak{D}\|_{0}+\|\mathcal{D}\|_{0}\right)^{1 / 2}+\frac{1}{\left(\|\mathfrak{D}\|_{0}+\|\mathcal{D}\|_{0}\right)^{1 / 2}} \frac{1}{l^{m}}\|\mathcal{D}\|_{0}\right) \leq C\left(\|\mathcal{D}\|_{0}^{1 / 2}+\frac{1}{l^{m}}\|\mathcal{D}\|_{0}^{1 / 2}\right)
\end{aligned}
$$

and hence achieves (5.15). Note that the scaling bound $(5.15)$ is consistent with the first requirement in (5.1) of Proposition 5.1.

3. Iterating the one-dimensional oscillations. We set $v_{1}=\mathfrak{v}, w_{1}=\mathfrak{w}$ and inductively define $v_{k+1} \in \mathcal{C}^{3}(\bar{\Omega})$ and $w_{k+1} \in \mathcal{C}^{2}\left(\bar{\Omega}, \mathbb{R}^{2}\right)$ for $k=1,2,3$ by means of Proposition 5.1 applied to $v_{k}, w_{k}$, the function $a_{k}$ and the unit vector $\xi_{k}$ appearing in 5.14, with the parameters:

$$
l_{k}=\frac{l}{\sigma^{k-1}}<1, \quad \lambda_{k}=\frac{1}{l_{k+1}}>\frac{1}{l_{k}},
$$

and with the remaining three parameters:

$$
\delta_{3} \geq \delta_{2} \geq \delta_{1}=\max _{m=1,2}\left\{l^{m}\|\nabla \mathfrak{v}\|_{m}\right\}+\max _{m=0 \ldots 3, k=1 \ldots 3}\left\{l^{m}\left\|a_{k}\right\|_{m}\right\}
$$

as indicated below. We then finally set: $\tilde{v}=v_{4}$ and $\tilde{w}=w_{4}$.

We start by checking that the assumptions of Proposition 5.1 are satisfied. Namely, we claim that $\delta_{k}, l_{k} \in(0,1)$ together with:

$$
\left\|a_{k}\right\|_{m} \leq \frac{\delta_{k}}{l_{k}^{m}} \quad \forall m=0 \ldots 3 \quad \text { and } \quad\left\|\nabla v_{k}\right\|_{m} \leq \frac{\delta_{k}}{l_{k}^{m}} \quad \forall m=1,2,
$$

at each iteration step $k=1,2,3$, if only the constant $\delta_{0}$ in (5.5) is appropriately small.

Indeed, $\delta_{1} \leq C\|\mathcal{D}\|_{0}^{1 / 2}$ in view of 5.11 and 5.15 , so $\delta_{1}<1$ if only $\delta_{0} \ll 1$. Further, by the definition 5.17 it follows that: $\left\|a_{k}\right\|_{m}=\frac{1}{l^{m}} l^{m}\left\|a_{k}\right\|_{m} \leq \frac{\delta_{1}}{l^{m}} \leq \frac{\delta_{k}}{l_{k}^{m}}$, so the first assertion in 
(5.18) holds. For the second assertion, we see directly that it holds when $k=1$, as: $\left\|\nabla v_{1}\right\|_{m}=$ $\frac{1}{l^{m}} l^{m}\|\nabla \mathfrak{v}\|_{m} \leq \frac{\delta_{1}}{l^{m}}$. On the other hand, using induction on $k$ and exploiting (5.3), we get:

$$
\begin{aligned}
\left\|\nabla v_{k+1}\right\|_{m} & \leq\left\|\nabla v_{k}\right\|_{m}+\left\|\nabla v_{k+1}-\nabla v_{k}\right\|_{m} \leq \frac{\delta_{k}}{l_{k}^{m}}+C \delta_{k} \lambda_{k}^{m} \\
& \leq \delta_{k}\left(\frac{1}{l_{k+1}^{m}}+\frac{C}{l_{k+1}^{m}}\right)=C \frac{\delta_{k}}{l_{k+1}^{m}} \leq \frac{\delta_{k+1}}{l_{k+1}^{m}} \quad \forall m=1,2 \quad \forall k=1,2 .
\end{aligned}
$$

The proof of (5.18) is now complete for the choice $\delta_{k+1}=C \delta_{k}$, where $C>1$ is, as always, an appropriately large universal constant. Consequently: $\delta_{2}, \delta_{3} \leq C\|\mathcal{D}\|_{0}^{1 / 2}<1$ if only $\delta_{0} \ll 1$.

4. We now directly verify the concluding estimates of Proposition 5.2. We have, in view of the definition of $\mathfrak{D}^{\prime}$ and (5.14):

$$
\begin{aligned}
\tilde{\mathcal{D}} & =A-\mathfrak{A}+\mathfrak{D}^{\prime}+\left(\frac{1}{2} \nabla v_{1} \otimes \nabla v_{1}+\operatorname{sym} \nabla w_{1}\right)-\left(\frac{1}{2} \nabla v_{4} \otimes \nabla v_{4}+\operatorname{sym} \nabla w_{4}\right) \\
& =A-\mathfrak{A}-\sum_{k=1}^{3}\left(\left(\frac{1}{2} \nabla v_{k+1} \otimes \nabla v_{k+1}+\operatorname{sym} \nabla w_{k+1}\right)-\left(\frac{1}{2} \nabla v_{k} \otimes \nabla v_{k}+\operatorname{sym} \nabla w_{k}+a_{k} \xi_{k} \otimes \xi_{k}\right)\right),
\end{aligned}
$$

and thus by $5.10,5.2$ and the definition of $l$, there follows 5.7 :

$$
\begin{aligned}
\|\tilde{\mathcal{D}}\|_{0} & \leq\|A-\mathfrak{A}\|_{0}+C \sum_{k=1}^{3} \frac{\delta_{k}^{2}}{\lambda_{k} l_{k}} \leq C\left(l^{\beta}\|A\|_{0, \beta}+\delta_{3}^{2} \sum_{k=1}^{3} \frac{1}{\lambda_{k} l_{k}}\right) \\
& \leq C\left(\frac{\|\mathcal{D}\|_{0}^{\beta / 2}}{M^{\beta}}\|A\|_{0, \beta}+3 \frac{\delta_{3}^{2}}{\sigma}\right) \leq C\left(\frac{\|\mathcal{D}\|_{0}^{\beta / 2}}{M^{\beta}}\|A\|_{0, \beta}+\frac{1}{\sigma}\|\mathcal{D}\|_{0}\right) .
\end{aligned}
$$

We now check (5.8), using (5.10), 5.13) and (5.4):

$$
\begin{aligned}
\|\tilde{v}-v\|_{1} & \leq\|\mathfrak{v}-v\|_{1}+\sum_{k=1}^{3}\left\|v_{k+1}-v_{k}\right\|_{1} \leq C\|\mathcal{D}\|_{0}^{1 / 2}+C \sum_{k=1}^{3} \delta_{k} \leq C\|\mathcal{D}\|_{0}^{1 / 2} \\
\|\tilde{w}-w\|_{1} & \leq\|\mathfrak{w}-w\|_{1}+\left\|\mathfrak{w}^{\prime}-\mathfrak{w}\right\|_{1}+\sum_{k=1}^{3}\left\|w_{k+1}-w_{k}\right\|_{1} \\
& \leq C\left(\|\mathcal{D}\|_{0}^{1 / 2}+\|\mathcal{D}\|_{0}+\sum_{k=1}^{3} \delta_{k}\left(1+\left\|\nabla v_{k}\right\|_{0}\right)\right) \leq C\|\mathcal{D}\|_{0}^{1 / 2}\left(1+\sum_{k=1}^{3}\left\|\nabla v_{k}\right\|_{0}\right) \\
& \leq C\|\mathcal{D}\|_{0}^{1 / 2}\left(1+\|\nabla v\|_{0}+\|\mathfrak{v}-v\|_{1}+\sum_{k=1}^{2}\left\|v_{k+1}-v_{k}\right\|_{1}\right) \\
& \leq C\|\mathcal{D}\|_{0}^{1 / 2}\left(1+\|\nabla v\|_{0}+\|\mathcal{D}\|_{0}^{1 / 2}\right) \leq C\|\mathcal{D}\|_{0}^{1 / 2}\left(1+\|\nabla v\|_{0}\right) .
\end{aligned}
$$

Finally, the first bound in 5.9 follows by 5.11 and 5.3 :

$$
\begin{aligned}
\|\tilde{v}\|_{2} & \leq\|\mathfrak{v}\|_{2}+\sum_{k=1}^{3}\left\|v_{k+1}-v_{k}\right\|_{2} \leq \frac{C}{l}\|\mathcal{D}\|_{0}^{1 / 2}+C \sum_{k=1}^{3} \delta_{k} \lambda_{k} \\
& \leq \frac{C}{l}\|\mathcal{D}\|_{0}^{1 / 2}+C \delta_{3} \sum_{k=1}^{3} \frac{\sigma^{k}}{l} \leq \frac{C}{l}\|\mathcal{D}\|_{0}^{1 / 2}\left(1+\sigma^{3}\right) \leq C M \sigma^{3}
\end{aligned}
$$


while the second bound is obtained by:

$$
\begin{aligned}
\|\tilde{w}\|_{2} & \leq\|\mathfrak{w}\|_{2}+\left\|\mathfrak{w}^{\prime}-\mathfrak{w}\right\|_{2}+\sum_{k=1}^{3}\left\|w_{k+1}-w_{k}\right\|_{2} \leq C\left(M+\|\mathcal{D}\|_{0}+\sum_{k=1}^{3} \delta_{k} \lambda_{k}\left(1+\left\|\nabla v_{k}\right\|_{0}\right)\right) \\
& \leq C\left(M+\delta_{3} \sum_{k=1}^{3} \frac{\sigma^{3}}{l}\left(1+\left\|\nabla v_{k}\right\|_{0}\right)\right) \leq C M\left(1+\sigma^{3}+\sigma^{3} \sum_{k=1}^{3}\left\|\nabla v_{k}\right\|_{0}\right) \\
& \leq C M \sigma^{3}\left(1+\sum_{k=1}^{3}\left\|\nabla v_{k}\right\|_{0}\right) \leq C M \sigma^{3}\left(1+\|\nabla v\|_{0}\right) .
\end{aligned}
$$

in view of (5.12), 5.13) and reasoning as in 5.19.

\section{The $\mathcal{C}^{1, \alpha}$ Approximations - a Proof of Theorem 1.2 .}

We are now in a position to state the final intermediary approximation result, parallel to [10, Theorem 1].

Theorem 6.1. Assume that $\Omega \subset \mathbb{R}^{2}$ is an open, bounded domain. Given are functions $v \in \mathcal{C}^{2}(\bar{\Omega})$, $w \in \mathcal{C}^{2}\left(\bar{\Omega}, \mathbb{R}^{2}\right)$ and $A \in \mathcal{C}^{0, \beta}\left(\bar{\Omega}, \mathbb{R}_{\text {sym }}^{2 \times 2}\right)$ for some $\beta \in(0,1)$, such that the deficit $\mathcal{D}$ below is appropriately small:

$$
\mathcal{D}=A-\left(\frac{1}{2} \nabla v \otimes \nabla v+\operatorname{sym} \nabla w\right), \quad 0<\|\mathcal{D}\|_{0}<\delta_{0} \ll 1 .
$$

Fix the exponent:

$$
0<\alpha<\min \left\{\frac{1}{7}, \frac{\beta}{2}\right\}
$$

Then, there exist $\bar{v} \in \mathcal{C}^{1, \alpha}(\bar{\Omega})$ and $\bar{w} \in \mathcal{C}^{1, \alpha}\left(\bar{\Omega}, \mathbb{R}^{2}\right)$ such that:

$$
\begin{gathered}
\frac{1}{2} \nabla \bar{v} \otimes \nabla \bar{v}+\operatorname{sym} \nabla \bar{w}=A, \\
\|\bar{v}-v\|_{1} \leq C\|\mathcal{D}\|_{0}^{1 / 2} \quad \text { and } \quad\|\bar{w}-w\|_{1} \leq C\left(1+\|\nabla \tilde{v}\|_{0}\right)\|\mathcal{D}\|_{0}^{1 / 2},
\end{gathered}
$$

where $C>0$ is a constant depending on $\alpha$ but independent of all other parameters.

Proof. The exact solution to 6.3 will be obtained as the $\mathcal{C}^{1, \alpha}$ limit of sequences of successive approximations $\left\{v_{k} \in \mathcal{C}^{2}(\bar{\Omega}), w_{k} \in \mathcal{C}^{2}\left(\bar{\Omega}, \mathbb{R}^{2}\right)\right\}_{k=1}^{\infty}$.

1. Induction on stages. We set $v_{0}=v$ and $w_{0}=w$. Given $v_{k}$ and $w_{k}$, define $v_{k+1}$ and $w_{k+1}$ by applying Proposition 5.2 with parameters $\sigma$ and $M_{k}$ that will be appropriately chosen below and that satisfy:

$$
M_{k}>\max \left\{\left\|v_{k}\right\|_{2},\left\|w_{k}\right\|_{2}, 1\right\} \quad \text { and } \quad \sigma>1 .
$$

Following our notational convention, we define the $k$-th deficit $\mathcal{D}_{k}=A-\left(\frac{1}{2} \nabla v_{k} \otimes \nabla v_{k}+\operatorname{sym} \nabla w_{k}\right)$. In view of Proposition 5.2, we get:

$$
\begin{gathered}
\left\|\mathcal{D}_{k+1}\right\|_{0} \leq C\left(\frac{\|A\|_{0, \beta}}{M_{k}^{\beta}}\left\|\mathcal{D}_{k}\right\|_{0}^{\beta / 2}+\frac{1}{\sigma}\left\|\mathcal{D}_{k}\right\|_{0}\right), \\
\left\|v_{k+1}-v_{k}\right\|_{1} \leq C\left\|\mathcal{D}_{k}\right\|_{0}^{1 / 2} \quad \text { and } \quad\left\|w_{k+1}-w_{k}\right\|_{1} \leq C\left(1+\left\|\nabla v_{n}\right\|_{0}\right)\left\|\mathcal{D}_{k}\right\|_{0}^{1 / 2},
\end{gathered}
$$




$$
\left\|v_{k+1}\right\|_{2} \leq C M_{k} \sigma^{3} \quad \text { and } \quad\left\|w_{k+1}\right\|_{2} \leq C\left(1+\left\|\nabla v_{k}\right\|_{0}\right) M_{k} \sigma^{3},
$$

provided that (5.5) holds for each $\mathcal{D}_{k}$. We shall now validate this requirement, with the parameters:

$$
M_{k}=\left(\mathfrak{C}\left(1+\left\|\nabla v_{0}\right\|_{0}\right) \sigma^{3}\right)^{k} M_{0}
$$

In fact, we will inductively prove that one can have:

$$
\left\|\mathcal{D}_{k}\right\|_{0} \leq \frac{1}{\sigma^{s k}}\|\mathcal{D}\|_{0} \quad \text { with any } \quad 0<s<\min \left\{1, \frac{6 \beta}{2-\beta}\right\} .
$$

Fix $s$ as indicated in (6.10). Clearly, (6.10) and 6.5$)$ hold for $k=0$. By (6.6) and the induction assumption we obtain the bound:

$$
\sigma^{s(k+1)} \frac{\left\|\mathcal{D}_{k+1}\right\|_{0}}{\|\mathcal{D}\|_{0}} \leq \frac{C\|A\|_{0, \beta}\|\mathcal{D}\|_{0}^{\beta / 2-1} \sigma^{s}}{M_{0}^{\beta}} \frac{1}{\mathfrak{C}^{k \beta}}\left(\frac{\sigma^{(1-\beta / 2)\left(s-\frac{6 \beta}{2-\beta}\right)}}{\left(1+\left\|\nabla v_{0}\right\|_{0}\right)^{\beta}}\right)^{k}+C \sigma^{s-1} .
$$

We see that in view of the condition on $s$ in 6.10 , both $\sigma^{s-1}$ and $\sigma^{(1-\beta / 2)\left(s-\frac{6 \beta}{2-\beta}\right)}$ are smaller than 1. Further, it is possible to choose $\sigma>1$ so that the second term in (6.11) be smaller than $1 / 2$ and that the quotient term in parentheses above is also smaller than 1 . Then, choose $M_{0}$ so that (6.5) holds for $k=0$ together with:

$$
\frac{C\|A\|_{0, \beta}\|\mathcal{D}\|_{0}^{\beta / 2-1} \sigma^{s}}{M_{0}^{\beta}}<\frac{1}{2} .
$$

This results in the first term in 6.11 being smaller than $1 / 2$ if only $\mathfrak{C} \geq 1$. Consequently, we get $\sigma^{s(k+1)}\left\|\mathcal{D}_{k+1}\right\|_{0} /\|\mathcal{D}\|_{0} \leq 1$ as needed in 6.10.

Observe now that by (6.7) and by the established (6.10):

$$
\begin{aligned}
\forall k \geq 0 \quad\left\|\nabla v_{k}\right\|_{0} & \leq\left\|\nabla v_{0}\right\|_{0}+\sum_{i=0}^{k-1}\left\|v_{i+1}-v_{i}\right\|_{1} \leq\left\|\nabla v_{0}\right\|_{0}+C \sum_{i=0}^{k-1}\left\|\mathcal{D}_{i}\right\|_{0}^{1 / 2} \\
& \leq\left\|\nabla v_{0}\right\|_{0}+C\left(\sum_{i=0}^{\infty} \frac{1}{\sigma^{s i / 2}}\right)\|\mathcal{D}\|_{0}^{1 / 2}=\left\|\nabla v_{0}\right\|_{0}+\frac{C}{1-\sigma^{-s / 2}}\|\mathcal{D}\|_{0}^{1 / 2} \\
& \leq\left\|\nabla v_{0}\right\|_{0}+C\|\mathcal{D}\|_{0}^{1 / 2},
\end{aligned}
$$

if only, say, $\sigma^{s}>4$ which can be easily achieved through the choice of $\sigma$. Now, by (6.8) and (6.12):

$$
\frac{\left\|v_{k+1}\right\|_{2}}{M_{k+1}} \leq \frac{1}{\mathfrak{C}} \frac{C}{\left(1+\left\|\nabla v_{0}\right\|_{0}\right)} \quad \text { and } \quad \frac{\left\|w_{k+1}\right\|_{2}}{M_{k+1}} \leq \frac{1}{\mathfrak{C}} \frac{C\left(1+\left\|\nabla v_{k}\right\|_{0}\right)}{\left(1+\left\|\nabla v_{0}\right\|_{0}\right)} \leq \frac{1}{\mathfrak{C}} \frac{C\left(1+\left\|\nabla v_{0}\right\|_{0}+\|\mathcal{D}\|_{0}^{1 / 2}\right)}{\left(1+\left\|\nabla v_{0}\right\|_{0}\right)} .
$$

Hence, taking the constant $\mathfrak{C} \gg 1$ large enough, we see that both quantities above can be made smaller than 1, proving therefore the required (6.5).

2. $\mathcal{C}^{1, \alpha}$ control of the approximating sequences $v_{n}$ and $w_{n}$. Let now $\alpha$ be an exponent as in 6.2. Choose $s$ satisfying 6.10 and:

$$
\alpha(6+s)-s<0
$$

It is an easy calculation that $s$ satisfying $(6.10)$ and $(6.13)$ exists if and only if the exponent $\alpha$ is in the range 6.2 . Indeed, $(6.13)$ is equivalent to $\alpha<\frac{s}{6+s}$, while 6.10 is equivalent to:

$$
0<\frac{s}{6+s}<\min \left\{\frac{1}{7}, \frac{\beta}{2}\right\}
$$


We will prove that sequences $\left\{v_{k}, w_{k}\right\}_{k=0}^{\infty}$ are Cauchy in $\mathcal{C}^{1, \alpha}(\bar{\Omega})$. Firstly, by 6.7), 6.12, 6.10):

$$
\begin{aligned}
\left\|v_{k+1}-v_{k}\right\|_{1} & \leq C\left\|\mathcal{D}_{k}\right\|_{0}^{1 / 2} \leq \frac{C}{\sigma^{s k / 2}}\|\mathcal{D}\|_{0}^{1 / 2} \\
\left\|w_{k+1}-w_{k}\right\|_{1} & \leq C\left(1+\left\|\nabla v_{k}\right\|_{0}\right)\left\|\mathcal{D}_{k}\right\|_{0}^{1 / 2} \leq \frac{C}{\sigma^{s k / 2}}\left(1+\left\|\nabla v_{0}\right\|_{0}+\|\mathcal{D}\|_{0}^{1 / 2}\right)\|\mathcal{D}\|_{0}^{1 / 2}
\end{aligned}
$$

so we see right away that they are Cauchy in $\mathcal{C}^{1}(\bar{\Omega})$. On the other hand, by $\left.(6.8), 6.12,66.10\right)$ :

$$
\left\|v_{k+1}-v_{k}\right\|_{2}+\left\|w_{k+1}-w_{k}\right\|_{2} \leq C\left(1+\left\|\nabla v_{k}\right\|_{0}\right) M_{k} \sigma^{3} \leq C\left(1+\left\|\nabla v_{0}\right\|_{0}+\|\mathcal{D}\|_{0}^{1 / 2}\right)\left(\mathfrak{C}\left(1+\left\|\nabla v_{0}\right\|_{0}\right) \sigma^{3}\right)^{k} M_{0},
$$

so the sequences have the tendency to diverge in $\mathcal{C}^{2}(\bar{\Omega})$. Interpolating now the $\mathcal{C}^{1, \alpha}$ norm by [1]:

$$
\|f\|_{0, \alpha} \leq\|f\|_{1}^{\alpha}\|f\|_{0}^{1-\alpha}
$$

we obtain:

$$
\begin{aligned}
\left\|\nabla\left(v_{k+1}-v_{k}\right)\right\|_{0, \alpha}+\left\|\nabla\left(w_{k+1}-w_{k}\right)\right\|_{0, \alpha} & \leq C_{0}^{\alpha}\left(C_{0} \sigma^{3}\right)^{k \alpha} M_{0}^{\alpha} \cdot C_{0}^{1-\alpha} \frac{1}{\sigma^{s k(1-\alpha) / 2}} \\
& =C_{0} M_{0}^{\alpha}\left(C_{0}^{\alpha}\right)^{h}\left(\sigma^{\frac{\alpha(6+s)-s}{2}}\right)^{k}
\end{aligned}
$$

where by $C_{0}$ we denoted an upper bound of all quantities involving $C, v_{0}, \mathcal{D}$. It is clear that choosing $\sigma$ sufficiently large (so that $C_{0} \sigma^{3-s / 2}<1$ ), the resulting bound 6.15 implies that $\left\{\nabla v_{k}, \nabla w_{k}\right\}_{k=0}^{\infty}$ are Cauchy in $\mathcal{C}^{0, \alpha}(\bar{\Omega})$, provided that $(6.13)$ holds. We see that the choice of exponent range in 6.2 so that the above construction technique works, is optimal.

3. Concluding, we see that $\left\{v_{k}, w_{k}\right\}_{k=0}^{\infty}$ converge to some $\bar{v} \in \mathcal{C}^{1, \alpha}(\bar{\Omega})$ and $\bar{w} \in \mathcal{C}^{1, \alpha}\left(\bar{\Omega}, \mathbb{R}^{2}\right)$. Since the defects in the approximating sequence obeys: $\lim _{k \rightarrow \infty}\left\|\mathcal{D}_{k}\right\|_{0}=0$ by $(6.10)$, we immediately get 6.3. Additionally, by 6.14):

$$
\begin{aligned}
\|\bar{v}-v\|_{1} & \leq \sum_{k=0}^{\infty}\left\|v_{k+1}-v_{k}\right\|_{1} \leq C\left(\sum_{k=0}^{\infty} \frac{1}{\sigma^{s k / 2}}\right)\|\mathcal{D}\|_{0}^{1 / 2}=\frac{C}{1-\sigma^{-s / 2}}\|\mathcal{D}\|_{0}^{1 / 2} \leq C\|\mathcal{D}\|_{0}^{1 / 2} \\
\|\bar{w}-w\|_{1} & \leq \sum_{k=0}^{\infty}\left\|w_{k+1}-w_{k}\right\|_{1} \leq C\left(\sum_{k=0}^{\infty} \frac{1}{\sigma^{s k / 2}}\right)\left(1+\|\nabla v\|_{0}\right)\|\mathcal{D}\|_{0}^{1 / 2} \leq C\left(1+\|\nabla v\|_{0}\right)\|\mathcal{D}\|_{0}^{1 / 2} .
\end{aligned}
$$

completing the proof of 6.4 .

We are now ready to give:

\section{Proof of Theorem 1.2.}

Fix a sufficiently small $\varepsilon>0$. We will construct $\bar{v} \in \mathcal{C}^{1, \alpha}(\bar{\Omega})$ and $\bar{w} \in \mathcal{C}^{1, \alpha}\left(\Omega, \mathbb{R}^{2}\right)$ such that:

$$
A_{0}=\frac{1}{2} \nabla \bar{v} \otimes \nabla \bar{v}+\operatorname{sym} \nabla \bar{w} \quad \text { in } \bar{\Omega}
$$

and:

$$
\left\|\bar{v}-v_{0}\right\|_{0}+\left\|\bar{w}-w_{0}\right\|_{0}<\varepsilon .
$$

In order to apply Theorem 6.1, we need to decrease the deficit $A_{0}-\left(\frac{1}{2} \nabla v_{0} \otimes \nabla v_{0}+\operatorname{sym} \nabla w_{0}\right)$ so that it obeys (6.1). This will be done in three steps. 
First, let $\tilde{v}_{0} \in \mathcal{C}^{\infty}(\bar{\Omega}), \tilde{w}_{0} \in \mathcal{C}^{\infty}\left(\Omega, \mathbb{R}^{2}\right)$ and $\tilde{A}_{0} \in \mathcal{C}^{\infty}\left(\bar{\Omega}, \mathbb{R}_{\text {sym }}^{2 \times 2}\right)$ be such that:

$$
\begin{aligned}
& \left\|\tilde{v}_{0}-v_{0}\right\|_{1}+\left\|\tilde{w}_{0}-w_{0}\right\|_{1}+\left\|\tilde{A}_{0}-A_{0}\right\|_{0}<\varepsilon^{2} \\
& \exists \tilde{c}_{0}>0 \quad A_{0}-\left(\frac{1}{2} \nabla \tilde{v}_{0} \otimes \nabla \tilde{v}_{0}+\operatorname{sym} \nabla \tilde{w}_{0}\right)>\tilde{c}_{0} \operatorname{Id}_{2} \quad \text { in } \bar{\Omega} .
\end{aligned}
$$

Second, by Theorem 2.1 and Remark 3.3 , there exists $v \in \mathcal{C}^{1}(\bar{\Omega})$ and $w \in \mathcal{C}^{1}\left(\Omega, \mathbb{R}^{2}\right)$ such that:

$$
\begin{array}{ll}
\tilde{A}_{0}=\frac{1}{2} \nabla v \otimes \nabla v+\operatorname{sym} \nabla w & \text { in } \bar{\Omega}, \\
\left\|v-\tilde{v}_{0}\right\|_{0}+\left\|w-\tilde{w}_{0}\right\|_{0}<\varepsilon^{2} & \text { and } \quad\left\|\nabla v-\nabla \tilde{v}_{0}\right\|_{0} \leq C .
\end{array}
$$

Third, let $\tilde{v} \in \mathcal{C}^{2}(\bar{\Omega})$ and $\tilde{w} \in \mathcal{C}^{2}\left(\Omega, \mathbb{R}^{2}\right)$ be such that:

$$
\|v-\tilde{v}\|_{1}+\|w-\tilde{w}\|_{1}<\varepsilon^{2} .
$$

By (6.19), 6.20) and 6.18), we get:

$$
\begin{aligned}
\| A_{0}-\left(\frac{1}{2} \nabla \tilde{v}\right. & \otimes \nabla \tilde{v}+\operatorname{sym} \nabla \tilde{w}) \|_{0} \\
& \leq\left\|A_{0}-\tilde{A}_{0}\right\|_{0}+\left\|\left(\frac{1}{2} \nabla \tilde{v} \otimes \nabla \tilde{v}+\operatorname{sym} \nabla \tilde{w}\right)-\left(\frac{1}{2} \nabla v \otimes \nabla v+\operatorname{sym} \nabla w\right)\right\|_{0} \\
& \leq\left\|A_{0}-\tilde{A}_{0}\right\|_{0}+\left(\|\nabla v\|_{0}+\|\nabla \tilde{v}\|_{0}\right)\|\nabla v-\nabla \tilde{v}\|_{0}+\|\nabla w-\nabla \tilde{w}\|_{0} \\
& \leq \varepsilon^{2}+\left(2\left\|\nabla v_{0}\right\|_{0}+2 \varepsilon^{2}+C\right) \varepsilon^{2}+\varepsilon^{2}<\delta_{0},
\end{aligned}
$$

as required in Theorem 6.1, if only $\varepsilon$ is small enough. We now apply Theorem 6.1 to $\tilde{v}, \tilde{w}$ and the original field $A_{0}$, and get $\bar{v} \in \mathcal{C}^{1, \alpha}(\bar{\Omega})$ and $\bar{w} \in \mathcal{C}^{1, \alpha}\left(\Omega, \mathbb{R}^{2}\right)$ satisfying $(6.16)$ and such that:

$$
\begin{aligned}
\left\|\bar{v}-v_{0}\right\|_{0}+\left\|\bar{w}-w_{0}\right\|_{0} & \leq C\left(1+\|\nabla \tilde{v}\|_{0}\right)\left\|A_{0}-\left(\frac{1}{2} \nabla \tilde{v} \otimes \nabla \tilde{v}+\operatorname{sym} \nabla \tilde{w}\right)\right\|_{0}+3 \varepsilon^{2} \\
& \leq C\left(1+\varepsilon^{2}+\left\|\nabla v_{0}\right\|_{0}\right)^{2} \varepsilon^{2}+3 \varepsilon^{2},
\end{aligned}
$$

by (6.4), 6.21, 6.20, 6.19) and 6.18). Clearly (6.17) follows, if $\varepsilon$ is small enough.

The following Corollary is of independent interest:

Corollary 6.2. Let $\Omega, f, p, \alpha$ be as in the statement of Theorem 1.1. Let $q \geq 2$. Then, for all $v_{0} \in W^{1, q}(\Omega)$, there exists a sequence $v_{n} \in \mathcal{C}^{1, \alpha}(\bar{\Omega})$ weakly converging to $v_{0}$ in $W^{1, q}(\Omega)$, and such that: $\operatorname{Det} \nabla^{2} v_{n}=f$ in $\Omega$.

Proof. Let $\bar{v}_{n} \in \mathcal{C}^{1}(\bar{\Omega})$ converge to $v_{0}$ in $W^{1, q}(\Omega)$. For every $\bar{v}_{n}$, consider the approximating sequence $\left\{v_{n, k} \in \mathcal{C}^{1, \alpha}(\bar{\Omega})\right\}_{k=1}^{\infty}$ as in Theorem 1.1. converging uniformly to $\bar{v}_{n}$. Define now $\left\{v_{n}\right\}$ to be an appropriate diagonal sequence, so that it converges to $v_{0}$ in $L^{q}(\Omega)$. We will check that $\left\{v_{n}\right\}$ is bounded in $W^{1, q}$.

The boundedness of $\left\|v_{n}\right\|_{L^{q}}$ is clear from the convergence statement. On the other hand, the proof of Theorem 1.2 gives, by (6.4), 6.18), 6.19), (6.20) and 6.21):

$$
\left|\nabla v_{n}(x)\right| \leq\left|\nabla \bar{v}_{n}(x)\right|+2 \epsilon^{2}+C+C \delta_{0}^{1 / 2} \leq\left|\nabla \bar{v}_{n}(x)\right|+C \quad \forall x \in \Omega .
$$

Consequently, $\left\|\nabla v_{n}\right\|_{L^{q}} \leq\left\|\nabla \bar{v}_{n}\right\|_{L^{q}}+C \leq C$, which concludes the proof. 


\section{Rigidity ReSults For $\alpha>2 / 3$ - A PRoof of TheOrem 1.3 .}

The crucial element in the proof of the rigidity Theorems 1.3 and 1.4 is the following result, that is the 'small slope analogue' of [10, Proposition 6]:

Proposition 7.1. Let $\Omega \subset \mathbb{R}^{2}$ be an open, bounded, simply connected domain. Assume that for some $\alpha \in(2 / 3,1)$, the function $v \in \mathcal{C}^{1, \alpha}(\bar{\Omega})$ is a solution to:

$$
\text { Det } \nabla^{2} v=f \quad \text { in } \bar{\Omega} \text {, }
$$

where $f \in L^{p}(\Omega)$ and $p>1$. Then the following degree formula holds true, for every open subset $U$ compactly contained in $\Omega$ and every $g \in L^{\infty}\left(\mathbb{R}^{2}\right)$ with supp $g \subset \mathbb{R}^{2} \backslash \nabla v(\partial U)$ :

$$
\int_{U}(g \circ \nabla v) f=\int_{\mathbb{R}^{2}} g(y) \operatorname{deg}(\nabla v, U, y) \mathrm{d} y .
$$

Above, $\operatorname{deg}(\psi, U, y)$ denotes the Brouwer degree of a continuous function $\psi: \bar{U} \rightarrow \mathbb{R}^{2}$ at a point $y \in \mathbb{R}^{2} \backslash \psi(\partial U)$.

Proof. 1. Fix $U$ and $g$ as in the statement of the Proposition. We first recall [35] that $\operatorname{deg}(\nabla v, U, \cdot)$ is well defined on the open set $\mathbb{R}^{2} \backslash \nabla v(\partial U)$. In fact, this function is constant on each connected component $\left\{U_{i}\right\}_{i=0}^{\infty}$ of $\mathbb{R}^{2} \backslash \nabla v(\partial U)$ and it equals 0 on the only unbounded component $U_{0}=$ $\mathbb{R}^{2} \backslash \nabla v(\bar{U})$. Thus, without loss of generality, we may assume that $g$ is compactly supported and that: supp $g \subset \bigcup_{k=1}^{\infty} U_{k}$. By compactness, there must be: supp $g \subset \bigcup_{k=1}^{N} U_{k}$ for some $N$, and consequently the integral in the right hand side of $(7.1)$ is well defined.

Let now $\left\{g_{i} \in \mathcal{C}_{c}^{\infty}\left(\bigcup_{k=1}^{N} U_{k}\right)\right\}_{i=1}^{\infty}$ be a sequence pointwise converging to $g$ and such that $\left\|g_{i}\right\|_{0} \leq$ $\|g\|_{L^{\infty}}$ for all $i$. It is sufficient to prove the formula (7.1) for each $g_{i}$ and pass to the limit by dominated convergence theorem. To simplify the notation, we drop the index, and so in what follows we are assuming that $g \in \mathcal{C}_{c}^{\infty}\left(\left(\mathbb{R}^{2} \backslash \nabla v(\partial U)\right) \cap \nabla v(\bar{U})\right)$.

As in the proof of Theorem 1.1, let $A \in W^{2, p}(\Omega) \cap \mathcal{C}^{0, \beta}(\bar{\Omega})$ be such that $\operatorname{curlcurl} A=-f$. Here, we take $\beta=\min \left\{2-\frac{2}{p}, \alpha\right\} \in(0,1)$. Consequently, in view of the simple connectedness of $\Omega$, there exists $w \in \mathcal{C}^{1, \beta}\left(\bar{\Omega}, \mathbb{R}^{2}\right)$ such that:

$$
A=\frac{1}{2} \nabla v \otimes \nabla v+\operatorname{sym} \nabla w .
$$

For a standard $2 \mathrm{~d}$ mollifier $\varphi \in \mathcal{C}_{c}^{\infty}(B(0,1))$ as in Lemma 4.3 , define:

$$
\forall l \in(0,1) \quad v_{l}=v * \varphi_{l}, \quad w_{l}=w * \varphi_{l}, \quad A_{l}=A * \varphi_{l},
$$

and apply the area formula (change of variable formula [14, 2]) to the smooth functions $g$ and $\nabla v_{l}$ :

$$
\int_{U}\left(g \circ \nabla v_{l}\right) \operatorname{det} \nabla^{2} v_{l}=\int_{\mathbb{R}^{2}} g(y) \operatorname{deg}\left(\nabla v_{l}, U, y\right) \mathrm{d} y .
$$

We see that $\nabla v_{l}$ converge uniformly to $\nabla v$, so the degrees converge pointwise [35] and thus:

$$
\lim _{l \rightarrow 0} \int_{\mathbb{R}^{2}} g(y) \operatorname{deg}\left(\nabla v_{l}, U, y\right) \mathrm{d} y=\int_{\mathbb{R}^{2}} g(y) \operatorname{deg}(\nabla v, U, y) \mathrm{d} y .
$$

To conclude the proof in view of $(7.2)$, it suffices now to show that:

$$
\lim _{l \rightarrow 0} \int_{U}\left(g \circ \nabla v_{l}\right) \operatorname{det} \nabla^{2} v_{l}=\int_{U}(g \circ \nabla v) f .
$$


2. Following [10, 9] we use a commutator estimate to get (7.3). As $f=-\operatorname{curl} \operatorname{curl} A$, we have:

$$
\begin{aligned}
& \left|\int_{U}\left(g \circ \nabla v_{l}\right) \operatorname{det} \nabla^{2} v_{l}-(g \circ \nabla v) f\right| \\
& \leq\left|\int_{U}\left(g \circ \nabla v_{l}\right)\left(\operatorname{det} \nabla^{2} v_{l}+\operatorname{curl} \operatorname{curl} A_{l}\right)\right|+\left|\int_{U}\left(g \circ \nabla v_{l}\right) \operatorname{curl} \operatorname{curl}\left(A_{l}-A\right)\right| \\
& \quad+\left|\int_{U}\left(\left(g \circ \nabla v_{l}\right)-(g \circ \nabla v)\right) f\right| .
\end{aligned}
$$

The second term above is bounded by $C \int_{U}\left|\nabla^{2} A_{l}-\nabla^{2} A\right| \leq C\left\|A_{l}-A\right\|_{W^{2, p}(\Omega)}$, hence it converges to 0 . The third term also converges to 0 by the dominated convergence theorem, since $g \circ \nabla v_{l}$ converges to $g \circ \nabla v$. In order to deal with the first term in (7.4), observe that det $\nabla^{2} v_{l}=$ $-\operatorname{curl} \operatorname{curl}\left(\frac{1}{2} \nabla v_{l} \otimes \nabla v_{l}+\operatorname{sym} \nabla w_{l}\right)$ and integrate by parts, in view of $g \circ \nabla v_{l}=0$ on $\partial U$ :

$$
\begin{aligned}
\mid \int_{U}\left(g \circ \nabla v_{l}\right) & \left(\operatorname{det} \nabla^{2} v_{l}+\operatorname{curl} \operatorname{curl} A_{l}\right) \mid \\
& =\left|\int_{U}\left\langle\nabla^{\perp}\left(g \circ \nabla v_{l}\right), \operatorname{curl}\left(\frac{1}{2} \nabla v_{l} \otimes \nabla v_{l}+\operatorname{sym} \nabla w_{l}-A_{l}\right)\right\rangle\right| \\
& \leq C\|\nabla g\|_{0}\left\|\nabla^{2} v_{l}\right\|_{0}\left\|\nabla v_{l} \otimes \nabla v_{l}-(\nabla v \otimes \nabla v) * \varphi_{l}\right\|_{1} \\
& \leq C \frac{1}{l^{1-\alpha}}\|\nabla v\|_{0, \alpha} \cdot \frac{1}{l^{1-2 \alpha}}\|\nabla v\|_{0, \alpha}^{2}=C \frac{1}{l^{2-3 \alpha}}\|\nabla v\|_{0, \alpha}^{3},
\end{aligned}
$$

where we used Lemma 4.3. Clearly, for $\alpha>2 / 3$ the right hand side in $(7.5)$ converges to 0 as $l \rightarrow 0$. By (7.4), this implies (7.3) and concludes the proof.

Below, we present all the details of the proof of Theorem 1.3. The proof of Theorem 1.4 will be postponed to [32].

\section{Proof of Theorem 1.3 .}

1. By Proposition 7.1 it follows that for all open sets $U \subset \bar{U} \subset \Omega$ :

$$
\operatorname{deg}(\nabla v, U, y)=0 \quad \forall y \in \mathbb{R}^{2} \backslash \nabla v(\partial U) .
$$

We would like to conclude [39, 40] that the image set $\nabla v(U)$ is of measure 0 . This will result in the developability of $v$, by the main statement of [28]. However, we note that there exist a Hölder continuous vector field whose local degree vanishes everywhere, but whose image is onto the unit square [33. Therefore, we will additionally exploit the gradient structure of $\nabla v$, using ideas of [27, Chapter 2], in combination with the commutator estimate technique as in the proof of Proposition 7.1.

Let $v_{l}=v * \varphi_{l}$ be as in the proof of Proposition 7.1 and for every $\delta>0$ define:

$$
u_{l, \delta}\left(x_{1}, x_{2}\right)=\nabla v_{l}\left(x_{1}, x_{2}\right)+\delta\left(-x_{2}, x_{1}\right), \quad u_{\delta}\left(x_{1}, x_{2}\right)=\nabla v\left(x_{1}, x_{2}\right)+\delta\left(-x_{2}, x_{1}\right) .
$$

Fix an open set $U$ with smooth boundary and compactly contained in $\Omega$. Let $g \in \mathcal{C}_{c}^{\infty}\left(\left(\mathbb{R}^{2} \backslash\right.\right.$ $\nabla v(\partial U)) \cap \nabla v(\bar{U}))$, and use the change of variable formula to $g$ and $u_{l, \delta}$ :

$$
\int_{U}\left(g \circ u_{l, \delta}\right)\left(\operatorname{det} \nabla^{2} v_{l}+\delta^{2}\right)=\int_{\mathbb{R}^{2}} g(y) \operatorname{deg}\left(u_{l, \delta}, U, y\right) \mathrm{d} y,
$$


where we noted that $\operatorname{det} \nabla u_{l, \delta}=\operatorname{det} \nabla^{2} v_{l}+\delta^{2}$. The integral in the right hand side of (7.7) is well defined for sufficiently small $l$ and $\delta$, because then $y \in \operatorname{supp} g$ implies $y \notin u_{l, \delta}(\partial U)$.

Passing to the limit, we immediately obtain:

$$
\lim _{l \rightarrow 0} \int_{\mathbb{R}^{2}} g(y) \operatorname{deg}\left(u_{l, \delta}, U, y\right) \mathrm{d} y=\int_{\mathbb{R}^{2}} g(y) \operatorname{deg}\left(u_{\delta}, U, y\right) \mathrm{d} y,
$$

while the left hand side of 7.7$)$ can be estimated by:

$$
\left|\int_{U}\left(g \circ u_{l, \delta}\right)\left(\operatorname{det} \nabla^{2} v_{l}+\delta^{2}\right)-\left(g \circ u_{\delta}\right) \delta^{2}\right| \leq\left|\int_{U}\left(g \circ u_{l, \delta}\right) \operatorname{det} \nabla^{2} v_{l}\right|+\left|\int_{U}\left(g \circ u_{l, \delta}-g \circ u_{\delta}\right) \delta^{2}\right| .
$$

The second term above clearly converges to 0 as $l \rightarrow 0$, because $u_{l, \delta}$ converge to $u_{\delta}$. The first term also converges to 0 as $\alpha>2 / 3$, where we reason exactly as in (7.4) and (7.5), keeping in mind that $f=0$. We hence conclude:

$$
\lim _{l \rightarrow 0} \int_{U}\left(g \circ u_{l, \delta}\right)\left(\operatorname{det} \nabla^{2} v_{l}+\delta^{2}\right)=\int_{U}\left(g \circ u_{\delta}\right) \delta^{2} .
$$

In view of $(7.8)$ and $(7.7)$ this implies:

$$
\forall 0<\delta \ll 1 \quad \int_{U}\left(g \circ u_{\delta}\right) \delta^{2}=\int_{\mathbb{R}^{2}} g(y) \operatorname{deg}\left(u_{\delta}, U, y\right) \mathrm{d} y .
$$

Consequently:

$$
\forall 0<\delta \ll 1 \quad \forall y \in u_{\delta}(U) \backslash u_{\delta}(\partial U) \quad \operatorname{deg}\left(u_{\delta}, U, y\right) \geq 1 .
$$

2. We now claim that:

$$
\nabla v(U) \subset \nabla v(\partial U)
$$

To prove (7.10) we argue by contradiction, assuming that for some $x_{0} \in U$ there is: $y_{0}=\nabla v\left(x_{0}\right) \in$ $\nabla v(U) \backslash \nabla v(\partial U)$. We distinguish two cases:

(i) There exist sequences $\left\{x_{k} \in U\right\}_{k=1}^{\infty}$ and $\delta_{k} \rightarrow 0^{+}$as $k \rightarrow \infty$ such that $y_{0}=u_{\delta_{k}}\left(x_{k}\right)$ for all $k$. Note that for large $k$, there must be $y_{0} \notin u_{\delta_{k}}(\partial U)$ because $u_{\delta}$ converges to $\nabla v$ as $\delta \rightarrow 0$ and $y_{0} \notin \nabla v(\partial U)$. In view of $(7.9)$ we get: $\operatorname{deg}\left(u_{\delta_{k}}, U, y_{0}\right) \geq 1$ contradicting (7.6).

(ii) For all $\delta$ small enough, $y_{0} \notin u_{\delta}(U)$. Thus $\operatorname{deg}\left(u_{\delta}, U, y_{0}\right)=0$, so by 7.9$)$ we get $y_{0} \in$ $u_{\delta}(\partial U) \subset \nabla v(\partial U)+C B(0, \delta)$, contradicting that $y_{0} \notin \nabla v(\partial U)$.

Our claim (7.10) is now established. Since the set $\nabla v(\partial U)$ is the image of a Hausdorff $1 \mathrm{~d}$ set $\partial U$ under a $\mathcal{C}^{0,1 / 2}$ deformation $\nabla v$, it has Lebesgue measure 0 (see [10, Lemma 4]). Thus $\nabla v(U)$ must have measure 0 for every smooth $U$ compactly contained in $\Omega$. The same then must be true for the entire set $\Omega$, i.e.: $|\nabla v(\Omega)|=0$ and we consequently obtain:

$$
\operatorname{Int}(\nabla v(\Omega))=\emptyset .
$$

3. By [29, Corollary 1.1.2.], condition (7.11) implies that every point $y \in \Omega$ has a convex open neighbourhood $\Omega_{y}$ such that for every point $x \in \Omega_{y}$ there is a line $L_{x}$ passing through $x$ so that $\nabla v$ is constant on $L_{x} \cap \Omega_{y}$. The same result in the present dimensionality has been first established in [28], see also footnote on pg. 875 in [29] for an explanation.

We now prove that $v$ is developable. Fix $x_{0} \in \Omega$ and let $[y, z] \subset \bar{\Omega}$ be the maximal segment passing through $x_{0}$ on which $\nabla v=\nabla v\left(x_{0}\right)$ is constant. Assume that $[y, z]$ does not extend to the 
boundary $\partial \Omega$, i.e. $y \in \Omega$. We will prove that then $\nabla v$ must be constant in an open neighbourhood of $x_{0}$. In fact, we will show that:

$$
V=\operatorname{Int}\left((\nabla v)^{-1}\left(\nabla v\left(x_{0}\right)\right)\right) \supset(y, z) .
$$

Let $(p, q)=L_{y} \cap \Omega_{y}$. By the maximality of $[y, z]$, the segment $(p, q)$ is not an extension of (is not parallel to) $[y, z]$. Also, $\nabla v=\nabla v\left(x_{0}\right)$ on $(p, q)$. Take any $y_{1} \in(y, z) \cap \Omega_{y}$ and define the open triangle $T=\operatorname{Int}\left(\operatorname{span}\left\{p, q, y_{1}\right\}\right)$. It is easy to notice that every line passing through any point $x \in T$ must intersect at least one of the segments $(p, q)$ or $\left(y, y_{1}\right)$. Since $T \subset \Omega_{y}$, it follows that $\nabla v(x)=\nabla v\left(x_{0}\right)$. Hence:

$$
\left(y, y_{1}\right) \subset T \subset V
$$

and, in particular, the set $V$ in 7.12 is nonempty.

To prove 7.12 assume, by contradiction, that there exists $y_{2} \in\left[y_{1}, z\right)$ so that:

$$
\left(y, y_{2}\right) \subset V \quad \text { but } \quad\left(y, y_{3}\right) \not \subset V \quad \forall y_{3} \in\left(y_{2}, z\right) .
$$

Now, the intersection $\Omega_{y_{2}} \cap V$ contains an open arc $C$ around the point $\left(y, y_{2}\right) \cap \Omega_{y_{2}}$. As above, we argue that every point in a sufficienty small open neighbourhood of the segment $I=(y, z) \cap \Omega_{y_{2}}$ must have the property that every line passing through it intersects $C$ or $I$, where $\nabla v=\nabla v\left(x_{0}\right)$. Consequently $I \subset V$, contradicting (7.13) and establishing (7.12).

\section{REFERENCES}

[1] R. Adams and J. Fournier, Sobolev Spaces, 2nd Edition, Academic Press 2003.

[2] L. Ambrosio, N. Fusco and D. Pallara, Functions of Bounded Variation and Free Discontinuity Problems, Oxford (2000).

[3] Yu. F. Borisov, The parallel translation on a smooth surface. III., Vestnik Leningrad. Univ. 14 (1959) no. 1, $34-50$.

[4] Yu. F. Borisov, Irregular surfaces of the class $\mathcal{C}^{1, \beta}$ with an analytic metric, (Russian) Sibirsk. Mat. Zh. 45 (2004), no. 1, 25-61; translation in Siberian Math. J. 45 (2004), no. 1, 19-52.

[5] T. Buckmaster, C. De Lellis, P. Isett, and L. Szekelyhidi, Jr., Anomalous dissipation for 1/5-Hölder Euler flows, Annals of Mathematics, 2015.

[6] T. Buckmaster, C. De Lellis, and L. Szekelyhidi, Jr., Transporting microstructures and dissipative Euler flows, to appear.

[7] T. Buckmaster, C. De Lellis and L. Szekelyhidi Jr., Dissipative Euler flows with Onsager-critical spatial regularity, Communications on Pure and Applied Mathematics, 2015.

[8] A. Choffrut and L. Szekelyhidi, Jr., Weak solutions to the stationary incompressible Euler equations, to appear in SIAM Journal of Mathematical Analysis.

[9] P. Constantin, W. E and E. S. Titi, Onsager's conjecture on the energy conservation for solutions of Euler's equation, Comm. Math. Phys. 165 (1994), no. 1, 207-209.

[10] S. Conti, C. De Lellis and L. Székelyhidi Jr., h-principle and rigidity for $\mathcal{C}^{1, \alpha}$ isometric embeddings, Proceedings of the Abel Symposium 2010.

[11] C. De Lellis, D. Inauen and L. Székelyhidi Jr., A Nash-Kuiper theorem for $C^{1, \frac{1}{5}-\delta}$ immersions of surfaces in 3 dimensions, to appear.

[12] C. De Lellis and L. Székelyhidi Jr., The Euler equations as a differential inclusion, Ann. of Math. (2) 170 (2009), no. 3, 1417-1436.

[13] C. De Lellis and L. Székelyhidi Jr., Dissipative continuous Euler flows, Invent. Math. 193 (2013), no. 2, $377-407$.

[14] L.C. Evans and R. Gariepy, Functions of Bounded Variation and Free Discontinuity Problems, CRC Press (1991).

[15] G.L. Eyink, Energy dissipation without viscosity in ideal hydrodynamics I. Fourier analysis and local energy transfer, Physica D: Nonlinear Phenomena 78, Issues 3-4, (1994), Pages 222-240. 
[16] I. Fonseca and J. Malý, From Jacobian to Hessian: distributional form and relaxation, Riv. Mat. Univ. Parma, 7, (2005), 4* (2005) 45-74.

[17] M. Gromov, Partial differential relations, Ergebnisse der Mathematik und ihrer Grenzgebiete (3) [Results in Mathematics and Related Areas (3)], 9. Springer-Verlag, Berlin, 1986.

[18] P. Isett, Hölder continuous Euler flows in three dimensions with compact support in time, to appear.

[19] P. Isett, Regularity in time along the coarse scale flow for the Euler equations, to appear.

[20] P. Isett and V. Vicol, Hölder continuous solutions of active scalar equations, to appear.

[21] T. Iwaniec, On the concept of weak Jacobian and Hessian, Report Univ. Jyväskylä 83 (2001), 181-205.

[22] R.L. Jerrard, Some remarks on Monge-Ampère functions, Singularities in PDE and the calculus of variations, CRM Proc. Lecture Notes, 44, (2008), 89-112.

[23] R.L. Jerrard, Some rigidity results related to Monge-Ampère functions, Canad. J. Math. 62, no. 2, (2010), 320-354.

[24] R.L. Jerrard and M.R. Pakzad, Sobolev spaces of isometric immersions of arbitrary dimension and codimension, Preprint (2014), http://arxiv.org/pdf/1405.4765v3.pdf

[25] S. Kim and B. Yan, Convex integration and infinitely many weak solutions to the Perona-Malik equation in all dimensions, to appear.

[26] S. Kim and B. Yan, Radial weak solutions for the Perona-Malik equation as a differential inclusion, to appear.

[27] B. Kirchheim, Geometry and Rigidity of Microstructures. Habilitation Thesis, Leipzig, (2001), Zbl pre01794210.

[28] M.V. Korobkov, Properties of the $\mathcal{C}^{1}$-smooth functions with nowhere dense gradient range, Sibirsk. Mat. Zh. 48 no. 6, (2007), 1272-1284; translation in Siberian Math. J. 48, no. 6, (2007), 1019-1028

[29] M.V. Korobkov, Properties of $\mathcal{C}^{1}$-smooth mappings with one-dimensional gradient range Sibirsk. Mat. Zh. 50 no. 5, (2009), 874-886; translation in Siberian Math. J. 50, no. 5, (2009), 874-886.

[30] N.H. Kuiper, On $\mathcal{C}^{1}$-isometric imbeddings. I, II., Nederl. Akad. Wetensch. Proc. Ser. A. 58, (1955) 545-556, 683-689.

[31] M. Lewicka, L. Mahadevan and M.R. Pakzad, The Monge-Ampère constraint: matching of isometries, density and regularity and elastic theories of shallow shells, to appear in Annales de l'Institut Henri Poincare (C) Non Linear Analysis.

[32] M. Lewicka and M.R. Pakzad, Rigidity and convexity of the very weak solutions to the Monge-Ampère equation, in preparation.

[33] Z. Liu and J. Malý, private communication.

[34] Z. Liu and M.R. Pakzad, Rigidity and regularity of co-dimension one Sobolev isometric immersions, to appear in Ann. Scuola Norm. Sup. Pisa Cl. Sci. (5).

[35] N. Lloyd, Degree theory, Cambridge University Press (1978).

[36] J. Nash, The imbedding problem for Riemannian manifolds, Ann. Math., 63, (1956), 20-63.

[37] J. Nash, $\mathcal{C}^{1}$ isometric imbeddings, Ann. Math., 60, (1954), 383-396.

[38] M.R. Pakzad, On the Sobolev space of isometric immersions, J. Differential Geom., 66, (2004) no. 1, $47-69$.

[39] A.V. Pogorelov, Surfaces with bounded extrinsic curvature (Russian), Kharhov, 1956.

[40] A.V. Pogorelov, Extrinsic geometry of convex surfaces, Translation of Mathematical Monographs vol. 35, American Math. Soc., 1973.

[41] V. Šverák, On regularity for the Monge-Ampère equation without convexity assumptions, preprint, HeriotWatt University (1991).

[42] L. Székelyhidi Jr., From isometric imbeddings to turbulence Max-Planck-Institut Lecture Notes (2013).

Marta Lewicka and Mohammad Reza Pakzad, University of Pittsburgh, Department of Mathematics, 139 University Place, Pittsburgh, PA 15260

E-mail address: lewicka@pitt.edu, pakzad@pitt.edu 